\title{
Die mittlere Durchflußmenge der Arterien des Menschen als Funktion des Gefäßradius.
}

\author{
Von \\ Prof. Dr. R. Thoma in Heidelberg. \\ (Mit 2 Textabbildungen.) \\ (Eingegangen am 1. April 1921.)
}

Meine Untersuchungen über das normale Wachstum und die pathologischen Umgestaltungen der Arterien des Menschen haben zu dem Schlusse genötigt, daß das zirkuläre Wachstum der Arterienlichtung in Abhängigkeit steht von der Geschwindigkeit der Randzonen des Blutstromes. Sodann führte die Prüfung der Stromgeschwindigkeiten in den Randzonen des Blutstromes zu bestimmten Ergebnissen bezüglich der mittleren Durchflußmengen und ihrer Beziehungen zu dem Mittelwert des Radius der Gefäßlichtung. Diese Ergebnisse sollen hier eine Zusammenfassung und Vervollständigung finden.

Die Durchflußmenge der Arterien ist veränderlich je nach dem Wechsel der Tätigkeit der Organe. Als mittlere Durchflußmenge aber kann man das für längere Zeiträume gebildete, arithmetische Mittel des Sekundenvolums bezeichnen, welches durch den Querschnitt einer Arterie strömt. Dagegen wäre ein arithmetisches Mittel für den Radius der lebenden Arterie schwer zu bilden, weil bereits bei der Bloßlegung die Lichtung der Arterie erhebliche Änderungen erfährt. Wenn man jedoch die Arterien unter den notwendigen Vorsichtsmaßregeln in situ der Leiche bei konstantem, den normalen Verhältnissen entsprechendem Drucke und bei annähernd normaler Temperatur mit Paraffin injiziert, so kann man mit bestimmten, früher besprochenen Hilfsmitteln ${ }^{1}$ ) die Radien der Gefäßlichtung verhältnismäßig genau messen und weiterhin annehmen, daß diese gemessenen Werte des Gefäßradius in einer bestimmten, gesetzmäßigen Beziehung zu den mittleren Durchflußmengen stehen. Zugleich entsteht jedoch die Aufgabe, diese Beziehung auf empirischem Wege genauer zu prüfen. Dabei macht man allerdings einige Voraussetzungen und namentlich die, daß der Druck in der aufsteigenden Aorta konstant und dem normalen Mitteldrucke gleich sei.

1) R. Thoma, Virchows Archiv 104. 1886; Beitr. z. pathol. Anat. u. z. allg. Pathol. 66. 1920. 
R. Thoma: Die mittlere Durchflußmenge der Arterien des Menschen usw. 283

Solche Voraussetzungen erscheinen jedoch als unvermeidlich, wenigstens gegenwärtig, wo die Untersuchung noch in ihren Anfängen steht.

Bezeichnet man den Radius der Gefäßlichtung mit $R$ und die Breite der plasmatischen, zellfreien Randzone des Blutstromes mit $\beta$, so wird, wie ich ${ }^{1}$ ) vor einer Reihe von Jahren gezeigt habe, unter Voraussetzung einer linearen; lamellären Strömung die Durchflußmenge $W$ einer Arterie in der Zeiteinheit gleich

$$
W=\frac{\pi}{2}\left[R^{2}+(R-\beta)^{2}+\frac{(R-\beta)^{4}}{2 n \beta\left(R-\frac{\beta}{2}\right)}\right] \varrho
$$

wenn man vorläufig der Einfachheit halber die Annahme macht, daß das Blut an der Gefäßwand adhäriert. Dabei bezeichnet $\varrho$ die Stromgeschwindigkeit an der Grenze des roten Axialstromes und der plasmatischen Randzone, welche Grenze dem Abstand $\beta$ von der Gefäßwand entspricht. Diese Stromgeschwindigkeit $\varrho$ ist jedoch gleich

$$
\varrho=\frac{1}{4 \vartheta}\left[R^{2}-(R-\beta)^{2}\right] \frac{d p}{d x}
$$

wenn $\vartheta$ den Viscositätskoeffizienten des Blutplasmas und der Ausdruck $\frac{d p}{d x}$ das Druckgefälle darstellt, welches gewöhnlich als Druck $p$ geteilt durch die Strombahnlänge $x$ bezeichnet wird.

Sodann gibt in Gleichung 1 die Größe $n$ das Verhältnis des Viscositätskoeffizienten des roten Axialstromes zu dem Viscositätskoeffizienten $\vartheta$ des Blutplasmas, so daß der Viscositätskoeffizient des roten Axialstromes gleich $n \vartheta$ wird. Die Größe $n$ kann indessen nicht unmittelbar mit dem Viscosimeter gefunden werden. Wenn man jedoch mit dem Viscosimeter den Viscositätskoeffizienten $\vartheta$ des Blutplasmas und den Viscositätskoeffizienten $\eta$ des Blutes bestimmt hat, so findet man, wie ich damals gezeigt habe, den Wert der Größe $n$ durch die Gleichung

$$
n=\frac{(R-\beta)^{4}}{\left(\frac{\vartheta}{\eta}-1\right) R^{4}+(R-\beta)^{4}}
$$

Solange nun die Koeffizienten $\vartheta$ und $\eta$ als konstante Größen zu betrachten sind, stimmt der Inhalt der Gleichungen 1 bis 3 vollständig mit dem Gesetze von $\mathrm{Hage} \mathbf{n}^{2}$ ) überein, welches sehr zu Unrecht nach Poise uille ${ }^{3}$ ) benannt wird und welches man schreiben kann

$$
W=\frac{\pi}{8 \eta} R^{4} \frac{d p}{d x}
$$

1) R. Thoma, Dtsch. Archiv f. klin. Med. 99. 1910.

2) Hage n, Pogg. Annalen der Physik u. Chemie 46. 1839.

3) Poiseuille, Mém. présentés par div. savants étrang. à l'acad. roy, des sc. de l'institut de France. Sc. math. et phys. Paris $\gamma .1841$; 9. 1846. 
Die Gleichungen 1-3 ermöglichen indessen, wie sich weiterhin zeigen wird, die Verfolgung einer großen Zahl von Beziehungen, welche durch die Gleichung 4 nicht aufgedeckt werden können ${ }^{1}$ ). Die oben gemachte Voraussetzung einer linearen, lamellären, wirbelfreien Strömung aber darf für den Blutstrom in den Arterien des Menschen unter normalen Bedingungen als erfüllt gelten, wie ich ${ }^{2}$ ) wiederholt besprochen habe. Meine Beweisführung stützte sich auf drei Tatsachen:

1. Die mittlere Stromgeschwindigkeit erreicht normalerweise in den Arterien des Menschen an keiner Stelle die Grenze, bei welcher nach den Untersuchungen von Hagenbach, Reynolds, Couette und Grüneisen der lineare, lamelläre Charakter der Strömung labil zu werden beginnt und die Entstehung von Stromwirbeln ermöglicht.

2. An den Verzweigungsstellen der kleinen Arterien und Venen zeigt das Mikroskop eine lineare, lamelläre, durchaus wirbelfreie Strömung. Man ist daher zu der Annahme berechtigt, daß die Innenfläche dieser Verzweigungsstellen genau die Gestalt der äußersten Lamelle einer sich teilenden, linearen Strömung besitzt. Die Gestalt der Verzweigungsstellen der großen Arterien ist jedoch der Gestalt der Verzweigungsstellen der kleinen Arterien geometrisch ähnlich, so daß kein Grund vorliegt, welcher zu der Annahme von Stromwirbeln berechtigen würde.

3. Die normale Blutströmung vollzieht sich in allen Arterien des lebenden Menschen geräuschlos. Unter pathologischen Bedingungen dagegen, bei welchen infolge abnorm geringer Viscosität oder abnorm großer Stromgeschwindigkeit des Blutes obige Grenze überschritten und Wirbelbildung ausgelöst wird, treten sofort Geräusche auf, welche mit dem Stethoskop hörbar sind.

In der Folge haben sich $\mathrm{Hess}^{3}$ ) und Hürthle ${ }^{4}$ ) dieser Argumentation angeschlossen. Sie führt zu dem Ergebnisse, daß der Blutstrom in den Arterien des normalen Menschen die Eigenschaften einer lamellären, linearen Strömung besitzt, linear genannt, weil die Widerstände des Stromes der ersten Potenz der Geschwindigkeit proportional sind.

Zugleich erweist es sich, daß die vorstehenden Entwicklungen bei der Untersuchung des normalen Blutkreislaufes des Menschen genau zutreffen für alle Arterien, welche mehr als 1,0 bis 1,5 mm Radius besitzen. Denn für diese größeren Arterien sind die Viscositätskoeffizienten $\vartheta$ des Blutplasma und $\eta$ des Blutes normalerweise hinreichend genau konstant, vorausgesetzt, daß man gebührende Rücksicht nimmt

1) Für den Fall, daß das Blut an der Gefäßwand gleitet, nehmen obige Gleichungen eine Form an, welche ich an dem früher genannten Orte gegeben habe.

2) R. Thoma, Dtsch. Archiv f. klin. Ned. 99. 1910; Zeitschr. f. experim. Pathol. u. Ther. 11. 1912.

3) W. R. Hess, Arch. f. d. ges. Physiol. 168, 474. 1917.

4) K. Hürthle, Ebenda 193, 159. 1918. 
auf die Temperatur und den Zellgehalt des Blutes. Außerdem ergibt sich, daß bei diesen größeren Arterien die Größe $n$ bei zunehmendem Radius der Gefäßlichtung nur eine geringe Abnahme erfährt, so daß für diese größeren Arterien $n$ ohne allzu großen Fehler als konstant betrachtet werden kann. Es hat dies seinen Grund in der geringen Größe des Wertes $\beta$, der Breite der plasmatischen Randzone.

1. Die Strömung des Blutes in engen und in weiten Röhren bei konstantem Druckgefälle.

Indessen haben bereits im Jahre $1906 \mathrm{~d} u$ Pré Denning und Watson $\left.{ }^{1}\right)$ durch eine große Untersuchungsreihe gezeigt, daß für Röhren von weniger als $1,5 \mathrm{~mm}$ Radius die Viscositätskoeffizienten $\vartheta$ des Blutplasmas und $\eta$ des Blutes nicht mehr konstant sind. Sie ändern sich bei gleichbleibendem Druckgefälle mit der Abnahme der Rohrlichtung und sie ändern sich bei gleichbleibender Rohrlichtung mit der Höhe des Druckgefälles. Hess ${ }^{2}$ ) und Rothmann ${ }^{3}$ ) haben diese Ergebnisse im wesentlichen bestätigt und zugleich von neuem gezeigt, daß unter den gleichen Bedingungen die Viscositätskoeffizienten nicht kolloider Flüssigkeiten, Wasser und verdünntes Glycerin konstant bleiben. Hess und Rothmann ziehen sodann aus ihren Ergebnissen den Schluß, daß das Gesetz von Hagen auf die Strömung von kolloiden Flüssigkeiten und Blut nicht anwendbar sei.

Nachweislich geht jedoch auch in engen Röhren der lineare Charakter der Blutströmung nicht verloren. Ich habe daher seinerzeit den Versuch gemacht, auf rein empirischem Wege Änderungen an obigen Gleichungen anzubringen, durch welche diese Gleichungen auf den Blutstrom in engen und weiten Röhren anwendbar werden. In diesem Sinne soll hier zunächst die Blutströmung in engen und weiten Röhren bei konstantem Druckgefälle besprochen werden.

Auf Grund der vorliegenden Beobachtungen kann man von der Vorstellung ausgehen, daß der Viscositätskoeffizient $n \vartheta$ des roten Axialstromes für engere Röhren größer ist, weil die plasmatische Randzone in engeren Röhren einen verhältnismäßig größeren Teil des Querschnittes der Rohrlichtung einnimmt, so daß die Blutzellen im Axialstrom dichter zusammengedrängt werden. Ein solches Verhältnis muß notwendigerweise den Viscositätskoeffizienten $n \vartheta$ des roten Axialstromes größer werden lassen, indem zugleich $n$ eine Funktion des Radius $R$ der Rohrlichtung wird. Damit steht man vor der Aufgabe, diese Funktion von $R$ zunächst auf empirischem Wege zu prüfen.

1) A. du Pré Denning and J. H. Watson, Proceedings of the Royal Society of London. Series B. 78, 328. 1906.

2) W. R. Hess, Arch. f. Physiol. 1912, S. 197; Arch. f. d. ges. Physiologie 162, 187. 1915.

3) M. Rothmann, Arch. f. d. ges. Physiol. 155, 318. 1914. 
Bei einem konstanten Druckgefälle, welches der Größenordnung nach ungefähr dem Druckgefälle in den kleinen Arterien des Menschen gleichkommt ${ }^{1}$ ), haben du Pré Denning und Watson die Viscositätskoeffizienten $\vartheta$ und $\eta$ des Blutplasmas und des Blutes in Glasröhren verschiedenen Kalibers bestimmt. Aus ihrer Tabelle $V$ findet man sodann, mit Hilfe der obigen Gleichung 3 und unter der Voraussetzung, da $\beta$ die Breite $\beta$ der plasmatischen Randzone des Blutes unter diesen Bedingungen in Glasröhren $0,0035 \mathrm{~mm}$ betrage:

$$
\begin{aligned}
& \text { für } R=1,0 \mathrm{~mm} \text { die Größe } n=2,0688 \\
& \text { für } R=0,5 \mathrm{~mm} \quad, \quad, \quad n=2,4949 \\
& \text { für } R=0,3 \mathrm{~mm} \quad, \quad, \quad, \quad n=3,1552 \\
& \text { für } R=0,15 \mathrm{~mm} \quad, \quad, \quad n=5,4120 \text {. }
\end{aligned}
$$

Man kann aus diesen Zahlen, welche bei einer Temperatur von $32^{\circ} \mathrm{C}$ für Pferdeblut mit 6 Mill. Zellen im Kubikmillimeter gelten, auf graphischem Wege eine Kurve ${ }^{2}$ ) gewinnen, welche diese Werte zum Ausdruck bringt unter der Voraussetzung, erstens da $B n$ für Werte von $R$, welche größer als $1 \mathrm{~mm}$ sind, nur noch eine geringe Abnahme erfährt, und zweitens daß $n$ für sehr kleine Werte von $R$ unendlich groß wird. Wenn die Lichtung des Rohres sehr enge wird, muß die Anwesenheit der roten Blutzellen bewirken, daß die verschiedenen Schichten des Axialstromes gleiche Geschwindigkeit annehmen, wobei $n=\infty$ wird. Es ist vorauszusehen, daß dieser Erfolg sicher erreicht wird, wenn der Durchmesser des roten Axialstromes nicht größer ist als der Druchmesser einer roten Blutzelle. In dem zellreichen Blute aber kann offenbar dieser Erfolg bereits in etwas weiteren Röhren eintreten.

Die auf graphischem Wege gewonnene Kurve besa $\$$, wie man bei den gegebenen Voraussetzungen leicht einsieht, ungefähr die Eigenschaften einer Hyperbel, deren Schenkel sich asymptotisch einer Abszisse und einer Ordinate näherten. Man kann jedoch auch auf rechnerischem Wege zu dem gleichen Ergebnisse gelangen, wenn man der Hyperbel die Form gibt:

1) Nur ausnahmsweise findet man bei den Autoren zuverlässige Anhaltspunkte zur Ermittelung des von ihnen benutzten Druckgefälles. Zumeist erfährt man nur einiges über den benützten Druck. Die Capillaren von du Pré Denning und Watson hatten ungefähr eine Iänge von $120 \mathrm{~mm}$. In diesen Capillaren strömte das Blut unter dem Úberdrucke einer Blutsäule, deren Höhe zu Anfang des Versuches ungefäbr $109 \mathrm{~mm}$ und am Fnde des Versuches ungefähr $91 \mathrm{~mm}$ betrug. Der mittlere Überdruck kann daher einer Blutsäule von $100 \mathrm{~mm}$ gleichgesetzt werden. In diesem Falle betrug nach der Umrechnung in Quecksilberdruck das Druckgefälle ungefähr 0,065. Für jeden Millimeter Strombahnlänge wurde der Druck von $0,065 \mathrm{~mm}$ hg verbraucht. Dies ist ein Druckgefälle, welches nach den später zu gebenden Gleichungen 15 und 16 in Arterien von ungefähr $0,5-0,2 \mathrm{~mm}$ Radius zu erwarten ist.

2) R. Thoma, Dtsch. Archiv f. klin. Med. 99, 586 und Kurve, Textabb. 2 auf S. 588. 1910 . 


$$
n=a+\frac{b}{R-c}
$$

In dieser Gleichung bezeichnen $a$ und $b$ zwei Konstante, die aus den Beobachtungen bestimmt werden sollen, und $\mathrm{c}$ entspricht demjenigen Werte des Radius $R$, für welchen $n$ unendlich groß wird. Auch dieser Wert $c$ kann aus den Beobachtungen berechnet werden.

Ich verfuhr dabei in der Weise, daß ich die oben zusammengestellten, mit Hilfe der Gleichung 3 gefundenen Werte von $n$ als beobachtete Werte betrachtete, welche von den variablen Werten von $R$ abhängig sind. Dann führt man in obiger Gleichung 5 zunächst für den Wert $c$, von dem man zufolge der Voraussetzungen, welche dieser Gleichung zugrunde liegen, wissen kann, daß er nicht sehr viel größer ist als der Durchmesser einer roten Blutzelle, zunächst eine ungefähr passende Größe, etwa $0,020 \mathrm{~mm}$ ein. Bezeichnet man sodann die variable Größe $(R-0,02)$ mit der neven Variablen $z$, so kann man weiterhin $\frac{1}{z}=v$ setzen, so $\operatorname{da} \beta$ obige Gleichung 5 die lineare Form

$$
n=a+b v
$$

annimmt. Nach Wittstein ${ }^{1}$ ) findet man in diesem Falle nach der Methode der kleinsten Fehlerquadrate die wahrscheinlichsten Werte der Konstanten $a$ und $b$ durch die Gleichungen:

$$
\begin{aligned}
a & =\frac{\Sigma\left(v^{2}\right) \Sigma(M)-\Sigma(v) \Sigma(v M)}{m \Sigma\left(v^{2}\right)-[\Sigma(v)]^{2}} \\
b & =\frac{m \Sigma(v M)-\Sigma(v) \Sigma(M)}{m \Sigma\left(v^{2}\right)-[\Sigma(v)]^{2}}
\end{aligned}
$$

wobei die einzelnen beobachteten Werte von $n$ mit $M$ bezeichnet werden und $m$ der Anzahl der Beobachtungen entspricht.

Das Ergebnis ist an die Voraussetzung geknüpft, daß $c=0,02 \mathrm{~mm}$ sei. Aus den wahrscheinlich sten Werten von $a$ und $b$ kann man sodann unter dieser Voraussetzung für die verschiedenen Werte von $R$ die zugehörigen Werte von $n$ berechnen. Vergleicht man diese mit den beobachteten Werten von $n$, so ergeben sich die Beobachtungsfehler $f$ und die Quadrate dieser Beobachtungsfehler führen endlich zu dem wahrscheinlichen Werte $g$ dieser Fehler, gleich

$$
g=0,6745 \sqrt{\frac{\Sigma\left(f^{2}\right)}{2}}
$$

Dieser wahrscheinliche Wert $g$ der Beobachtungsfehler ist zunächst für $c=0,02 \mathrm{~mm}$ ziemlich gro $B$ und zwar gleich 0,07385 . Wiederholt man jedoch die Rechnung für eine Reihe verschiedener kleinerer und größerer Werte von $c$, so findet man, daß diese wahrscheinlichen Beobachtungsfehler bei zunehmendem Werte von $c$ stetig kleiner werden, bis sie ein tiefes Minimum mit 0,001434 erreichen, wenn $c=0,052 \mathrm{~mm}$ gesetzt wird. Für jeden Wert von $c$, der kleiner oder größer als $0,052 \mathrm{~mm}$ ist, werden die wahrscheinlichen Beobachtungsfehler erheblich größer. Nimmt man $c=0,07$ so erreicht der wahrscheinliche Fehler bereits wieder den Wert 0,05036 .

(Der wahrscheinliche Fehler $g$ der gegebenen Beobachtungsreihe erscheint hier als eine Funktion von $c$, welche Funktion für $c=0,052 \mathrm{~mm}$ ein Minimum anfweist. Gleichzeitig erscheinen auch die Konstanten $a$ und $b$ als varjable Werte,

1) Th. Wittstein, Zusatz zu seiner deutschen Übersetzung von L. Navier, Lehrbuch der Differential- und Integralrechnung. Bd. 2. Hannover 1854. 
welche sich als Funktionen von $c$ darstellen. Doch entbehrt sowohl $a$ als $b$ eines Minimums. Mit steigenden Werten von $c$ nimmt $a$ stetig zu, während gleichzeitig $b$ mit steigenden Werten von $c$ stetig abnimmt.)

Für $c=0,052 \mathrm{~mm}$, welches dem Minimum des wahrscheinlichen Fehlers $g$ entspricht, werden dabei die wahrscheinlichsten Werte von $a=1,6813$ und von $b=0,36561$ gefunden. Nach den Lehren der Wahrscheinlichkeitsrechnung lautet daher die gesuchte Gleichung der Hyperbel

$$
n=1,6813+\frac{0,36561}{R-0,052} \frac{\mathrm{mm}}{10}
$$

Sodann stellen sich die beobachteten und die aus dieser Hyperbel berechneten Werte von $n$ wie folgt:

\begin{tabular}{c|c|c|c|c}
\hline $\begin{array}{c}R \\
\mathrm{~mm}\end{array}$ & $\begin{array}{c}n \\
\text { beobachtet }\end{array}$ & $\begin{array}{c}n \\
\text { berechnet }\end{array}$ & $\begin{array}{c}\text { Fehler } \\
-\end{array}$ & $\begin{array}{c}\text { Fehler } \\
+\end{array}$ \\
\hline 1,0 & 2,0688 & 2,0670 & 0,0018 & - \\
0,5 & 2,4949 & 2,4973 & - & 0,0024 \\
0,3 & 3,1553 & 3,1555 & - & 0,0002 \\
0,15 & 5,4120 & 5,4120 & 0,0000 & -
\end{tabular}

und der wahrscheinliche Wert der Beobaehtungsfehler wird, wie bereits oben bemerkt wurde, $=0,001434$, womit zugleich der Sorgfalt und Genauigkeit der Viscositätsmessungen von $d u$ Pré Denning und Watson eine gewichtige Anerkennung gewährt wird.

Aus der Hyperbelgleichung aber folgt, da $\beta n$ unendlich groß wird für $c=0,052 \mathrm{~nm}$. In einer Arterie von $0,052 \mathrm{~mm}$ Radius und ebenso in allen kleineren Arterien würde daher die Stromgeschwindigkeit in allen Zonen des roten Axialstromes gleichgroß sein. Fine gegenseitige Verschiebung der Flüssigkeitsschichten wäre in diesen sehr kleinen Arterien nur im Bereiche der plasmatischen, zellfreien Randzonen des Stromes anzunehmen. Die Hy perbelgleichung aber findet ihren graphischen Ausdruck in der Kurve I der später folgenden Textabb. 1.

Die Untersuchungen von du Pré Denning und Watson enthalten auf ihrer Tab. $V$ noch eine zweite und dritte Beobachtungsreihe, welche der gleichen Behandlung unterzogen werden können. Sie beriehen sich auf Pferdeblut, welches 3,6 Millionen Zellen im Kubikmillimeter enthielt, jedoch bei verschiedener Temperatur strömte.

Bei der zweiten Untersuchungsreihe betrug die Temperatur $40,4^{\circ} \mathrm{C}$ und die Rechnung nach der Methode der kleinsten Quadrate ergab für das 3,6 Millionen Zellen enthaltende Blut ein Minimum für den wahrscheinlichen Wert des Beobachtungsfehlers, sowie $c=0,029 \mathrm{~mm}$ gesetzt wurde. In diesem Falle fand sich:

$$
n=1,488 \mathrm{I}+\frac{0,17724}{R-0,029 \mathrm{~mm}}
$$

entsprechend der Kurve II auf Textabb. 1. Die beobachteten und die aus dieser Gleichung berechneten Werte von $n$ aber waren:

\begin{tabular}{c|c|c|c|c}
\hline $\begin{array}{c}R \\
\mathrm{~mm}\end{array}$ & $\begin{array}{c}n \\
\text { beobachtet }\end{array}$ & $\begin{array}{c}n \\
\text { berechnet }\end{array}$ & $\begin{array}{c}\text { Fehler } \\
-\end{array}$ & $\begin{array}{c}\text { Fehler } \\
+\end{array}$ \\
\hline \hline 1,0 & 1,6512 & 1,6706 & - & 0,0194 \\
0,5 & 1,9036 & 1,8644 & 0,0392 & - \\
0,3 & 2,1201 & 2,1421 & - & 0,0220 \\
0,15 & 2,9550 & 2,9529 & 0,0021 & -
\end{tabular}


Der wahrscheinliche Wert der Beobachtungsfehler ist hier beträchtlich größer $=0,02337$, offenbar infolge der größeren Schwierigkeiten der Beobachtung. GröBere Fehler sind hier zu erwarten, weil die Unterschiede der Viscositätskoeffizienten des Plasmas und des Blutes geringer sind.

Bemerkenswert aber ist, daß hier bei dem geringen Zellgehalte des Blutes nicht nur der Viscositätsfaktor $n$, sondern auch der Wert von $c$ beträchtlich kleiner wird als in der ersten Beobachtungsreihe. Die Grenze, an welcher der Viscositätsfaktor $n$ und folglich auch der Viscositätskoeffizient $n \boldsymbol{v}$ des roten Axialstromes unendlich groß wird, rückt hier in erheblich engere Arterien, in Arterien vom Radius $0,029 \mathrm{~mm}$ vor, ein Ergebnis, das von vornherein zu erwarten war.

Die dritte Beobachtungsreihe von $d u$ Pré Denning und Watson bezieht sich auf Pferdeblut mit 3,6 Millionen Zellen, welches bei $32,2^{\circ} \mathrm{C}$ strömte. Das Minimum für die wahrscheinlichen Beobachtungsfehler fand sich hier, als $c=0,021 \mathrm{~mm}$ gesetzt wurde und die Gleichung der Hyperbel wurde:

$$
n=1,3866+\frac{0,18026}{R-0,021 \mathrm{~mm}}
$$

entsprechend der Kurve III auf Textabb. 1. Die beobachteten und die berech. neten Werte von $n$ aber stellten sich wie folgt:

\begin{tabular}{c|c|c|c|c}
\hline \hline $\begin{array}{c}R \\
\mathrm{~mm}\end{array}$ & $\begin{array}{c}n \\
\text { beobachtet }\end{array}$ & $\begin{array}{c}n \\
\text { berechnet }\end{array}$ & $\begin{array}{c}\text { F e h } \\
\text { Fehler } \\
+\end{array}$ \\
\hline 1,0 & 1,5661 & $\mathbf{1 , 5 7 0 7}$ & - & 0,0046 \\
0,5 & 1,7668 & $\mathbf{1 , 7 6 2 9}$ & 0,0039 & - \\
0,3 & 2,0351 & $\mathbf{2 , 0 3 2 8}$ & 0,0023 & - \\
0,15 & 2,7822 & $\mathbf{2 , 7 8 3 9}$ & - & 0,0017
\end{tabular}

Aus den Fehlerquadraten ergab sich sodann der wahrscheinliche Wert des Fehlers für die einzelnen Beobachtungen $=0,003184$.

Aus der zweiten und dritten Beobachtungsreihe wird man den Schluß ziehen, daß sowohl der Viscositätsfaktor $n$ wie der Wert von $c$, bei welchem $n$ unendlich groß wird, bei abnehmender Temperatur kleiner werden. Dies ist leicht verständlich, wenn man erwägt, daß bei abnehmender Temperatur der Viscositätskoeffizient $\vartheta$ das Blutplasmas erheblich rascher zunimmt als der Viscositätskoeffizient $\eta$ des Blutes.

Bei der Beurteilung der Zuverlässigkeit dieser Zahlen wolle man beachten, daß jeweils zur Bestimmung von 2 Konstanten vier analoge Gleichungen zur Verfügung standen, indem die dritte Konstante $c$ durch Variation gefunden wurde. Die Zuverlässigkeit der Zahlen gewinnt jedoch weiterhin noch dadurch, daß die vorstehenden drei Beobachtungsreihen gut vergleichbare Werte von $n$ und $c$ ergeben, die durchaus den tatsächlichen Verhältnissen zu entsprechen scheinen.

Die unvermeidlichen Fehler der Kalibrierung der Viscosimeterröhren haben indessen hier keine erschöpfende Berücksichtigung erfahren. Sie konnten allerdings, namentlich wenn immer der gleiche Satz von Röhren Verwendung fand, die numerischen Ergebnisse in merklicher Weise beeinflussen. Doch scheint mir, hei sorgfältiger Erwägung aller Einzelheiten, eine wesentliche Änderung des Gesamtergebnisses ausgeschlossen zu sein. Spätere Untersuchungen werden jedenfalls diesen Punkt genauer zu berücksichtigen haben.

Eine vierte Beobachtungsreihe von $\mathrm{d} u \mathrm{Pr}$ é Denning und Watson bezieht sich auf Pferdeblut mit 9,6 Millionen Zellen im Kubikmillimeter und auf eine Temperatur von $32^{\circ} \mathrm{C}$. Diese Beobachtungsreihe ergibt indessen nur drei Werte von $n$. 


$$
\begin{array}{clcc}
\text { Für } & R=1,0 \mathrm{~mm} & \text { wird } & n=3,786 \\
" & R=0,5, " & , & n=4,423 \\
, & R=0,15, \quad & , & n=17,02
\end{array}
$$

Zur Bestimmung der drei Konstanten der Hyperbelgleichung 5 stehen somit nur 3 Gleichungen zur Verfügung, aus welchen man auf algebraischem Wege findet

$$
n=3,300+\frac{0,4280}{R-0,1188 \mathrm{~mm}}
$$

Diese Gleichung gibt selbstverständlich für die verschiedenen Werte von $R$ fehlerfrei die beobachteten Werte von $n$. Leider gewinnt man jedoch keinen Anhaltspunkt über die Größe der Beobachtungsfehler. Aus den drei früheren Beobachtungsreihen allerdings darf man schließen, daß auch hier mit der wünschenswerten Sorgfalt gearbeitet und ein annähernd richtiges Resultat erzielt wurde. Dem entspricht auch der Inhalt der Gleichung. Der Viscositätsfaktor $n$ des roten Axialstromes ist hier etwa in dem Grade erhöht, wie es bei dem sehr hohen Zellgehalt und bei der gegebenen Temperatur zu erwarten war und auch der Wert von $c$ hat eine entsprechende Erhöhung auf $0,119 \mathrm{~mm}$ erfahren. Man darf somit behaupten, daß auch diese vierte Beobachtungsreihe die oben gewonnenen Firgebnisse bestätigt.

Für manche Zwecke und namentlich für die Berechnung des Druckgefälles wird es jedoch notwendig, noch einige Vorstellungen über die Größe des Viscositätskoeffizienten $\vartheta$ de; Blutplasmas zu gewinnen. Ich versuche daher noch eine Ausgleichung der Beobachtungsfehler von $\vartheta$. Wenn das Blutplasma als kolloide Substanz, den gegenwärtigen Anschauungen entsprechend, aus festen und flüssigen Teilchen besteht, so erscheint es wahrscheinlich, daß auch für die Viscositätskoeffizienten $\vartheta$ ähnliche Gesetze bestehen wie für das aus Zellen und Plasına bestehende Blut. Man könnte in diesem Falle setzen

$$
\vartheta=a+\frac{b}{R-c}
$$

mit dem allerdings sehr gewichtigen Unterschiede, daß $c$ so klein ist, daß es gegenüber den Werten ron $R$ verschwindet. Damit vereinfacht sich die Gleichung und lautet jetzt

$$
\vartheta=a+\frac{b}{R}
$$

bei einem vorläufig ausreichenden Grade der Annäherung. Mit Hilfe der Methode der kleinsten Fehlerquadrate findet man sodann aus je vier zusammengehörigen Viscositätsmessungen der Tabelle $V$ von $d u$ Pré Denning und Watson.

$$
\text { für Pferdeblutplasma bei } 32,2^{\circ} \mathrm{C} \quad \vartheta=0,01327+\frac{0,00015029}{R}
$$

mit einem wahrscheinlichen Werte der Beobachtungsfehler $=0,0002891$ und

$$
\text { für Pferdeblutplasma bei } 40,4^{\circ} \mathrm{C} \quad \vartheta=0,01129+\frac{0,00014886}{R}
$$

mit einem wahrscheinlichen Werte des Beobachtungsfehlers $=0,0003088$. Dabei ergeben die numerischen Werte von $\vartheta$, welche aus diesen beiden Gleichungen hervorgehen, wenn $R$ in Millimetern ausgedrückt wird, die numerischen Werte des Viscositätskoeffizienten des Blutplasmas in dem von du Pré Denning und Watson benützten Maßsystem: Zentimeter, Gramm, Sekunde. Der oben behandelte Viscositätsfaktor $n$ dagegen ist, wie man leicht einsieht, unabhängig vom Maßsystem.

Aus den Viscosimetermessungen von du Pré De nning und Watson kann man, wie vorstehende Untersuchung zeigt, den Schluß ziehen, daß 
der Viscositätsfaktor $n$ des roten Axialstromes eine variable Größe darstellt, die abhängig ist von dem Radius der Rohrlichtung und von dem Zellgehalt und der Temperatur des Blutes. Dieses Abhängigkeitsverhältnis ist für Pferdeblut, welches 6 Millionen Zellen im Kubikmillimeter enthält und bei einer Temperatur von $32^{\circ} \mathrm{C}$ durch Glasröhren strömt, gegeben durch die Hyperbelgleichung

$$
n=1,6813+\frac{0,36561}{R-0,052 \mathrm{~mm}}
$$

wenn auch $R$ in Millimetern ausgedrückt ist. Zugleich findet sich der wahrscheinliche Wert der Beobachtungsfehler gleich 0,001434. Die Eigenschaften dieser Hyperbel ergeben sich in übersichtlicher Weise aus der Kurve I der Abb. 1, in welcher der Radius der Rohrlichtung

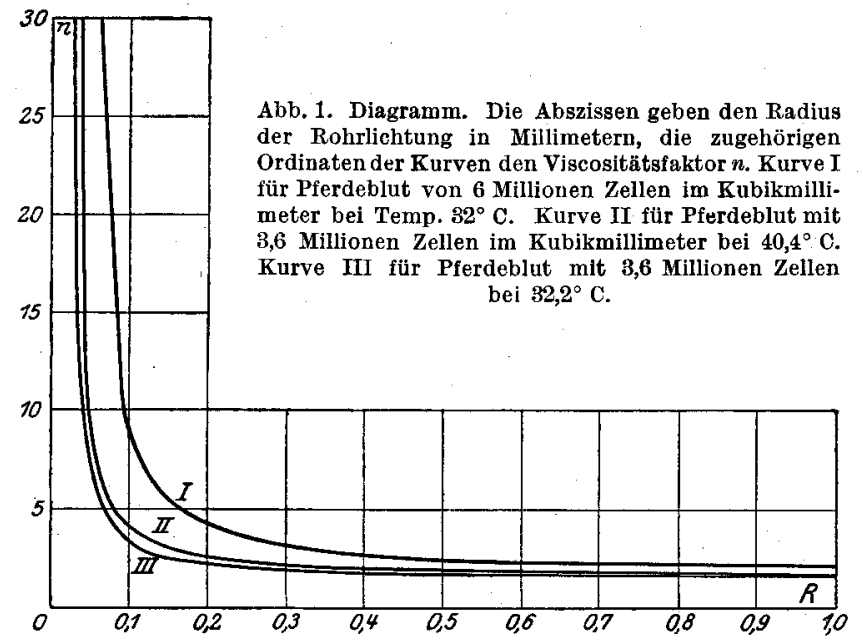

als Abszisse und der Viscositätsfaktor $n$ als Ordinate erscheint ${ }^{1}$ ). Für weitere Röhren ändert sich der Viscositätsfaktor $n$ nur in unerheblichem Maße. Sowie jedoch der Radius der Rohrlichtung unter den Wert von $1 \mathrm{~mm}$ herabgeht, wird $n$ rasch größer, um unendlich groß zu werden, wenn der Radius der Rohrlichtung gleich oder kleiner als $c=0,052 \mathrm{~mm}$ wird. In diesem Falle ist die Stromgeschwindigkeit in allen Zonen des roten Axialstromes gleich groß, und die Bewegung des strömenden Blutes vollzieht sich ausschließlich durch Verschiebungen der Flüssigkeitsschichten, welche die plasmatische Randzone des Blutes bilden.

Es ist dies ein Ergebnis, welches hier rein empirisch, ohne weitere Voraussetzungen aus den Viscositätsmessungen hervorgeht. Die Aus-

1) In Abb. 1 sind, wie früher (Dtsch. Arch. f. klin. Med. 99, 588. 1910), die Ordinaten, welche die Werte von $n$ darstellen, der Raumersparnis halber in kleinerem Maßstabe gezeichnet als die Radien $R$ der Rohrlichtung, welche als Abszissen dienen. 
rechnung hat zunächst gezeigt, daß die beobachteten Werte des Viscositätsfaktors $n$ mit großer Genauigkeit einer Hyperbelfunktion von $R$ entsprechen. Weiterhin aber wurde bei der Rechnung namentlich keine Voraussetzung über die Größe des Wertes von $c$ gemacht. Diese Größe $c$ wurde vielmehr ausschließlich durch die Anwendung der Methode der kleinsten Fehlerquadrate gefunden. Wäre $c$ gleich Null oder negativ, so würde die Rechnung dies notwendigerweise ergeben haben. Die Rechnung dagegen führte nicht nur in dieser ersten Beobachtungsreihe, sondern auch in drei weiteren Beobachtungsreihen zu gut stimmenden, positiven Werten von $c$. Es kann somit kein Zweifel darüber bestehen, daß von einer bestimmten Grenze an, welche durch die Größe $c$ angezeigt wird, in allen kleineren Arterien $n$ unendlich groß und die Stromgeschwindigkeiten in allen Teilen des roten Axialstromes unter sich gleich werden.

Dieses Ergebnis steht zugleich in voller Ubereinstimmung mit den Wahrnehmungen, welche man mit Hilfe des Mikroskopes an dem Blutstrom in den kleinen Gefäßen lebender Tiere macht. Hier dürften alle Zellen des roten Axialstromes mit gleicher Geschwindigkeit strömen, was allerdings in den Arterien erst deutlich erkennbar wird, wenn der Blutstrom durch lokale Störungen erheblich verzögert ist.

Es wäre sehr wünschenswert, Viscositätsbestimmungen, wie sie von du Pré Denning und Watson ausgeführt wurden, auch für menschliches Blutplasma und Blut von normaler Zusammensetzung und normaler Temperatur zu besitzen. Da jedoch solche Viscositätsmessungen nicht vorliegen, glaube ich vorläufig keinen allzu großen Fehler zu begehen, wenn ich für normal temperiertes und normal zusammengesetztes menschliches Blut die Werte von $n$ annehme, welche durch die Kurve I und die zugehörige Hyperbelgleichung zum Ausdrucke gebracht sind. Für normales menschliches Blut von normaler Körpertemperatur wäre demnach

$$
n=1,6813+\frac{0,3656}{R-0.052 \mathrm{~mm}}
$$

Das normale menschliche Blut enthält zwar in der Regel etwas weniger als 6 Millionen Zellen im Kubikmillimeter und strömt zumeist bei einer etwas höheren Temperatur als $32^{\circ} \mathrm{C}$. Nach den Erörterungen des Kleintextes, welche sich auf den Einfluß des Zellgehaltes und der Temperatur beziehen, bedingt geringerer Zellgehalt eine Verkleinerung des Faktors $n$ und höhere Temperatur eine Erhöhung dieses Faktors. Obige Annahme, welche in Gleichung 6 zum Ausdrucke gelangt, erscheint daher als gerechtfertigt, wenn sie auch nur auf eine annähernde Richtigkeit Anspruch erheben kann.

Die Vorstellung, welche man sich auf Grund dieser Untersuchung über die Stromgeschwindigkeiten in den verschiedenen Zonen eines Gefäß- 
querschnittes bilden kann, gelangt in übersichtlicher Weise zum Ausdruck durch zwei sich durchschneidende Umdrehungsparaboloide (Abb. 2). Das eine länger ausgezogene Paraboloid, welches auf dem axialen, in der Textabbildung gegebenen Längsschnitt als eine schlanke Parabel erscheint, gibt füx die Zeiteinheit die Stromgeschwindigkeiten im Bereiche der plasmatischen Randzone des Stromes. Die zweite, flacher gestaltete Parabel begrenzt dagegen die Stromgeschwindigkeiten in dem roten, hier grau getönten Axialstrom. Demgemäß gelangen die in einem gegebenen Zeitpunkt in der Ebene $\alpha-\alpha$ befindlichen Teile des Stromes, wie die Pfeile versinnlichen, nach Ablauf einer Zeiteinheit bis zu der krummen Fläche, welche im Randstrom von dem ersten Paraboloid und im Axialstrom von dem zweiten Paraboloid gegeben ist. Die Krümmung des zweiten Paraboloides aber wird mit zunehmendem Viscositätsfaktor $n$ des Axialstromes flacher und

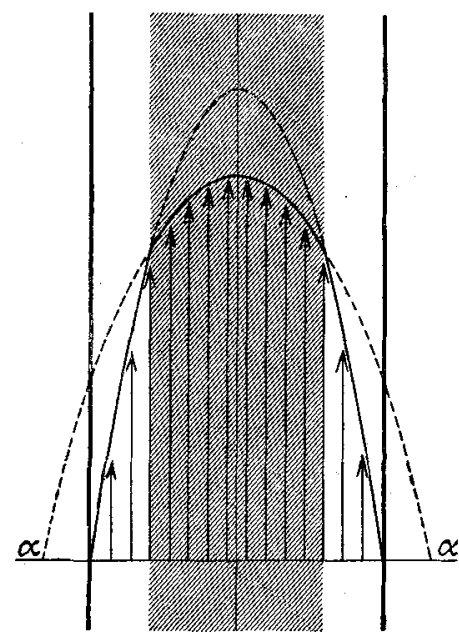

Abb. 2. Schema der Stromgeschwindigkeiten in dem plasmatischen Randstrom und in dem roten, hier grau dargestellten Axialstrom einer kleinen Arterie. flacher, bis sie für $n=\infty$ zu einer ebenen Fläche wird. Der Kubikinhalt des von der Ebene $\alpha-\alpha$ und den beiden Paraboloiden um. grenzten, hier mit Pfeilen ausgefüllten Raumes endlich gibt das Durchflußvolum in der Zeiteinheit.

Es sind dies theoretische Anschauungen, welche sich an die Gleichungen 1, 2, 3, 5, 6 knüpfen. Gegen diese Anschauungen macht Hess geltend, daß eine ähnliche Änderung der Viscosität in engen Röhren auch bei der Strömung zellfreier, kolloider Flüssigkeiten nachweisbar wird. Doch darf man aus dieser Tatsache nicht schließen, daß die hier vertretenen, theoretischen Anschaunngen irrige sind. Einerseits kann das Bestehen einer plasmatischen Randzone des Blutstromes nicht geleugnet werden und andererseits wäre es sehr wohl möglich, daß auch in strömenden, kolloiden, zellfreien Flüssigkeiten sich eine Randzone von geringer Viscosität bildet. Denn die kolloiden Flüssigkeiten werden, wenn sie auch homogen erscheinen, als eine Vereinigung flüssiger und fester Teilchen betrachtet, obgleich letztere nicht ohne besondere Hilfsmittel demonstriert werden können. Diese Anschauung hat in der Tat bereits oben (im Kleintext) eine befriedigende Anwendung gefunden bei der Prüfung der Viscosität des reinen Blutplasmas. Was aber den Deformationswiderstand kolloider Flüssigkeiten anbetrifft, so sind wir über seine Größe noch keineswegs genügend informiert. In den 
Gleichungen $1-6$ sind aber offenbar auch die Wirkungen des Deformationswiderstandes enthalten.

Vor allem aber ist zu betonen, daß diese Gleichungen auf empirischem Wege entstanden sind. Sie geben daher ganz unabhängig von jeder Theorie bei sachgemäßer Anwendung auf rein rechnerischem Wege annähernd genaue Werte für die Durchflußmengen. Sorgfältige Bestimmungen der Viscositätskoeffizienten $\vartheta$ des Blutplasmas und $\eta$ des Blutes des Menschen werden aber zweifellos die Genauigkeit der hier gegebenen Werte von $n$ noch erheblich zu steigern imstande sein.

Die hier gewonnenen Ergebnisse sind zugleich auch von großer Bedeutung für die Technik der Viscosimetrie. Offenbar ist es ganz unzulässig, die Zähigkeitskoeffizienten $\vartheta$ des Blutplasma, $n \vartheta$ des roten Axialstromes und $\eta$ des Blutes als konstante Werte zu behandeln und sie gleichmäßig auf große und kleine Blutgefäße und auf enge und weite Viscosimeterröhren anzuwenden. Die aus den Hyperbelgleichungen hervorgehenden Werte mögen mancherlei Ungenauigkeiten aufweisen, sie sind demungeachtet sehr viel näher der Wahrheit.

Für die Viscosimetrie am Krankenbette muß man dementsprechend für alle einzelnen Apparate gleiches und genau bekanntes Kaliber der Capillarröhren verlangen. Die beliebte Kontrolle der ärztlichen Viscosimeter mit Wasser hat nur dann einen Zweck, wenn zugleich das Kaliber der Viscosimeterröhre bekannt ist. Anderenfalls führt diese Kontrolle zu einer sehr gefährlichen, scheinbaren Exaktheit, zu unvergleichbaren Zahlen, wie sie in der Literatur vielfach hervortreten.

2. Die Strömung des Blutes in engen und weiten Röhren bei variablem Druckgefälle.

Du Pré Denning und Watson, Hess und Rothmann haben gezeigt, daß bei der Strömung des Blutes in engen Röhren die Durchflußmengen etwas rascher zunehmen als das Druckgefälle. Auch hier besteht somit eine Abweichung von dem Gesetze von Hagen. Man kann daher zunächst nach dem Grunde dieser Abweichung fragen.

Meines Erachtens dürfte dieser Grund, wie ich ${ }^{1}$ ) früher ausführte, gegeben sein in dem Verhalten der plasmatischen Randzone des strömenden Blutes. Bereits Poise uille ${ }^{2}$ ) bemerkte, daß die Breite der plasmatischen Randzone der Arterien bei abnehmender Stromgeschwindigkeit kleiner wird, um schließlich zu verschwinden, wenn der Blutstrom zum Stillstand gelangt. Ich kann diese Befunde für den Frosch wie für den Hund und das Kaninchen bestätigen, will jedoch hinzufügen, daß bei

1) R. Thoma, Deutsches Arch. f. klin. Med. 99, 1910.

2) Poiseuille, Mém. prés. par div. savants étrang. à l'acad. des sc. de l'institut de France. Sc. math. et phys. $\gamma, 105$. Paris 1841. 
abnehmender Stromgeschwindigkeit auch in den Arterien zunächst noch ein Zustand des Blutstromes eintritt, bei welchem die Leukocyten in größerer Zahl in die Randzone gelangen. Es ist dies die typische „Randstellung" der Leukocyten, welche Waller und Cohnheim in den Venen und Capillaren vorangehen sahen der Emigration der Leukocyten. Wie ich ${ }^{1}$ ) nachweisen konnte, tritt diese Randstellung der Leukocyten bei genügender Verlangsamung des Stromes in Arterien, Venen und Capillaren auch dann ein, wenn infolge einer geringen Wasserverarmung des Blutes die Lenkocyten ihre amöboiden Eigenschaften und damit die Fähigkeit der Emigration verlieren. Die Randstellung der Leukocyten ist somit ausschließlich Folge einer mäßigen Verzögerung des Blutstromes. Bei sehr hohen Stromgeschwindigkeiten endlich, welche bei der Durchschneidung von Arterien und Venen eintreten ${ }^{2}$ ), wird die plasmatische Randzone sehr viel breiter als normal.

Die Breite der plasmatischen Randzone nimmt in jeder Arterie mit der Stromgeschwindigkeit zu, wenn auch sehr erhebliche Steigerungen der Stromgeschwindigkeit erforderlich sind, um eine auffällige, leicht erkennbare Verbreiterung der Randzone zu erzeugen. Die Stromgeschwindigkeit aber steigt mit dem Druckgefälle. Es ist daher anzunehmen, daß bei steigendem Druckgefälle die Breite der plasmatischen Randzone um ein geringes zunimmt. Für die Durchflußmenge großer Arterien und für Glasröhren von mehr als 1,0 $\mathrm{mm}$ Radius würde dies von keiner nennenswerten Bedeutung sein. Dagegen würden, wie ich früher gezeigt habe, in den engeren Röhren die Durchflußmengen in Ubereinstimmung mit.der Beobachtung rascher zunehmen als das Druckgefälle.

Wenn man diese Frage weiter verfolgen will, kann man daher zunächst die Breite $\beta$ der plasmatischen Randzone betrachten als eine Funktion der Randstromgeschwindigkeit $\varrho$, welche an der Grenze der plasmatischen Randzone und des roten Axialstromes besteht. Dies scheint berechtigt zu sein, weil offenbar die Entstehung der plasmatischen Randzone abhängig ist von den Differenzen der Stromgeschwindigkeit der verschiedenen Lamellen des Stromes. Denn die Differenzen der Geschwindigkeit der Stromlamellen nehmen zu, wenn die Randstromgeschwindigkeit $\varrho$ eine Zunahme erfährt. Zwischen der Randstromgeschwindigkeit $\varrho$ und dem Druckgefälle $\frac{d p}{d x}$ bestehen sodann die Beziehungen, welche in der Gleichung 2 ihren Ausdruck gefunden haben.

1) R. Thoma, Der Einfluß des Wasser- und Salzgehaltes des Blutes und der Gewebssäfte auf die Auswanderung und auf die Form- und Ortsveränderungen farbloser Blutzellen. Virchows Archiv 62. 1874; y4. 1878.

2) Über die Technik solcher Versuche vgl. R. Thoma. Virchows Archiv 65. 1875. \%. 1878 . 
Wie ich bereits früher besprochen habe (Dtsch. Arch. f. klin. Med. 99. 1910), kann man auf Grund der Viscositätsmessungen der, beiden englischen Autoren den störenden Einfluß des ungleichen Druckgefälles eliminieren, wenn man für ein sehr zellreiches Blut die Breite $\beta$ der plasmatischen Randzone proportional der 7. bis 10. Wurzel der Randstromgeschwindigkeit @ annimmt. Man könnte daher schätzungsweise für normales Menschenblut $\beta$ proportional der 10 . Wurzel der Randstromgeschwindigkeit $c$ setzen.

Für konstante Randstromgeschwindigkeit $\varrho$ in dem konstanten Abstande $\beta$ von der Gefäßwand würde in diesem Falle der störende Einfluß ungleichen Druckgefälles in Wegfall kommen.

Es wird sich jedoch später zeigen, daß die Randstromgeschwindigkeit $\varrho$ in den kleinen Arterien des Menschen erheblich größer ist als in den großen Arterien, und zwar in einem Verhältnisse, welches weiterhin durch die auf empirischem Wege gewonnene Gleichung 14 gegeben wird. Wenn man sodann auf Grund der mikroskopischen Untersuchungen für eine Arterie von $0,028 \mathrm{~mm}$ Padius die Breite $\beta$ der plasmatischen Randzone $=0,01 \mathrm{~mm}$ annimmt, so kann man unter der Voraussetzung, da $\beta \beta$ der 10 . Wurzel der Randstronigeschwindigkeit $\varrho$ proportional ist, ohne Schwierigkeit die Breite $\beta$ der Randzone für die Arterien größeren und kleineren Kalibers ausrechnen. Sodann findet man auf graphischem Wege, daB diese Werte von $\beta$ annähernd auf einer fyperbel liegen und auf rechnerischem Wege ergibt sich annähernd

$$
\beta=0,006931+\frac{0,02190}{R+7,108} \text { Millimeter. }
$$

wobei auch $R$ in Millimetern einzusetzen ist. Aus dieser Gleichung aber folgt:

$$
\begin{aligned}
& \text { für } R=11,2 \mathrm{~mm} \text { (Aorta asc.) } \\
& \text {, } R=1 \text {, , } \\
& \text { " } R=0,1 \quad, \\
& \text {, } R=0,028, \\
& \text { " } \quad R=0,005, \\
& \begin{array}{l}
\beta=0,00813 \mathrm{~mm} \\
\beta=0,00963, \\
\beta=0,00997, \\
\beta=0,01000, \\
\beta=0,01001,
\end{array}
\end{aligned}
$$

Hier macht sich eine Besonderheit bemerkbar, welche mit der Beobachtung sehr gut in Übereinstimnuung steht. Die Breite $\beta$ der plasmatischen Randzone ist in allen der mikroskopischen Untersuchung zugängigen Arterien annähernd genau gleich groß, obgleich bei den größten dieser der mikroskopischen Untersuchung zugängigen Arterien der Radius der Lichtung ungefähr $20 \mathrm{mal}$ so groß ist als bei den kleinsten derselben. Zugleich erkennt man jedoch, daß aus der annähernd konstanten Breite der plasmatischen Randzone in den kleinsten Arterien kein Schluß gezogen werden darf auf die Breite der Randzone in den mittleren und größten Arterien.

Die weitere Rechnung aber zeigt, daß durch diese Annahme bezüglich des Wertes ron $\beta$ keine prinzipiellen Änderungen des Gesamtresultates erzielt werden, vorausgesetzt, daß man nunmehr diese Werte von $\beta$ als annähernd zutreffend annimmt und dann von neuem aus den vorhandenen Beobachtungen eine Gleichung für $\varrho$ sucht in der Weise, wie dies später besprochen werden soll. Die Gleichung für $\varrho$ gewinnt dabei etwas andere Konstanten, behält jedoch ihre allgemeine Form einer Exponentialfunktion. Zugleich erfahren die numerischen Werte für die Durchflußmengen, für das Druckgefälle und für die Geschwindigkeiten auf den versehiedenen Teilen des Stromquerschnittes nur sehr geringe, kaum nennenswerte Verschiebungen. Ich werde auf diese Änderungen später zurückkommen.

Eine Einführung dieser durch die ungleiche Randstromgeschwindigkeit bedingten Korrekturen wird erst gerechtfertigt sein, wenn eine ausreichende Zahl systematisch für diesen $Z$ weck, mit Röhren verschiedenen 
Kalibers angestellter Beobachtungen vorliegt. Gegenwärtig nimmt man dabei allerdings bei der Berechnung der mittleren Durchflußmengen der kleinen Arterien einen Fehler mit in den Kauf, der nicht ganz außer acht gelassen werden darf. Nach den Untersuchungen von du Pré Denning und Watson schien er 1\% nicht merklich zu übersteigen. Nach den Untersuchungen von Roth mann ist er jedoch beträchtlich größer. Hier liegen offenbar Beobachtungsfehler vor. Diese sind möglicherweise bei Rothmann zu suchen, der mit Strömen von stetig zunehmender Geschwindigkeit arbeitete, während die englischen Autoren an Strömungen von annähernd konstanter Geschwindigkeit beobachteten. Außerdem halte ich es für richtiger, mit senkrecht gestellten Capillaren zu untersuchen, weil in horizontal gestellten Capillaren immer eine Senkung der roten Zellen eintritt, welche die Zellen in ungleicher Weise auf dem Querschnitt des Stromes verteilt. Vorläufig möchten daher die Ergebnisse der englischen Autoren noch als maßgebend anzusehen sein.

Wenn jedoch Hess und Rothmann der von mir gegebenen Erklärung der Tatsachen entgegenhalten, daß in engen Röhren auch die Durchflußmengen zellfreier, kolloider Flüssigkeiten rascher wachsen als das Druckgefälle, so kann man diesem Einwand wiederum begegnen durch den Hinweis auf den Bau der kolloiden Flüssigkeiten. Wenn diese, wie man annimmt, aus festen und flüssigen Teilchen bestehen, so ist es sehr wohl denkbar, daß auch in kolloiden Flüssigkeiten bei der Strömung durch enge Röhren Randzonen geringerer Viscosität entstehen, welche bei höherem Druckgefälle breiter werden.

Für das strömende Blut jedoch ist das Vorhandensein einer zellfreien Randzone von variabler Breite eine feststehende Tatsache, so daß ein Vergleich des Blutes mit Lösungen von Gelatine und Stärke mir keine große Erleuchtung zu bringen scheint. Eher könnte meines Erachtens das Verhalten deś Blutes zur Erklärung dienen für das Verhalten dieser zellfreien, kolloiden Flüssigkeiten.

3. Die Strömung des Blutes in den Arterien des Menschen.

Die obigen Gleichungen 1, 2, 3, 5, 6 gewähren mit einiger Genauigkeit vollständige Auskunft über die numerischen Werte der mittleren Durchflußmengen der verschiedenen Arterien des Menschen, deren Radius größer als $1 \mathrm{~mm}$ ist, sowie entweder das Druckgefälle $\frac{d p}{d x}$ oder die Randstromgeschwindigkeit $\varrho$ in dem Abstande $\beta$ von der Gefäßwand für die verschiedenen Arterien bekannt ist. Die Kenntnis eines dieser Werte genügt, da zwischen beiden die Beziehungen bestehen, welche in der Gleichung 2 ihren Ausdruck fanden. Jede Annahme, die man entweder bezüglich des Druckgefälles $\frac{d p}{d x}$ oder der Randstrom- 
geschwindigkeit $\varrho$ macht, definiert daher einen bestimmten Bautypus des Arteriensystemes. Für die Arterien von weniger als $1 \mathrm{~mm}$ Radius werden dagegen mit Hilfe dieser Gleichungen die Durchflußmengen nur in mehr oder weniger annähernder Weise gefunden werden können.

Meine langjährigen Untersuchungen ${ }^{1}$ ) über die Entstehung, das Wachstum und die Erkrankungen der Blutgefäße haben, zum Teil mit der Schärfe des willkürlich herbeigeführten Experimentes zu dem Ergebnisse geführt, daß das Wachstum des Radius der Gefäßlichtung unabhängig ist von dem Blutdrucke, jedoch abhängig von der Stromgeschwindigkeit des Blutes. Eine Beziehung zwischen der Stromgeschwindigkeit und dem zirkulär gerichteten Wachstum der Gefäßwand ist jedoch am einfachsten erklärlich, wenn man annimmt, daß die Gefäßwand in irgendwelcher Weise die Geschwindigkeiten der Randzonen des Blutstromes empfindet und auf diese Empfindung in gesetzmäßiger Weise durch ein positives oder negatives Wachstum des Radius der Gefäßwand reagiert.

Wie dieses Wachstum sich vollzieht, habe ich an anderen Orten ausführlich besprochen, würde jedoch hier zu weit führen. Wenn aber die Gefäßwand die Geschwindigkeit der Randzonen des Blutstromes empfindet und auf diese Empfindung in gesetzmäßiger Weise reagiert, so ist es wiederum am einfachsten anzunehmen, da $\beta$ dieses positive oder negative Wachstum zur Folge hat, daß die Randstromgeschwindigkeit $\varrho$ in dem konstanten Abstande $\beta$ von der Gefäßwand gleichfalls konstant ist. Ich untersuche daher zunächst, wie sich der Blutstrom in dem Arteriensystem des Menschen darstellt, wenn für einen konstanten Wert von $\beta$ auch die Randstromgeschwindigkeit $\varrho$ konstant ist.

DaB durch eine solche Beziehung zwischen $\beta$ und $\varrho$ ein bestimmter Bautypus des Arteriensystems gegeben ist, geht bereits aus obigen Erörterungen hervor, die zeigen, daß damit das Druckgefälle und die Durchflußmengen für alle Arterien als Funktion von $R$ bestimmt sind: Es kommt jedoch darauf an zu prüfen, ob dieser Bautypus wirklich gegeben ist, weil auch andere Annahmen für $\varrho$ und $\beta$ gemacht werden können. Erst wenn dieser Bautypus empirisch nachgewiesen ist, darf

1) Die wichtigsten dieser Untersuchungen finden sich zusammengestellt in dem Dtsch. Arch. f. klin. Med. 99, 565. 1910. Seit jener Zeit kamen hinzu: R. Thoma, Über die Histomechanik des Gefäßsystems und die Pathogenese der Arteriosklerose. Virchows Archiv 204. 1911. - Die Strömung an den Verzweigungsstellen der Blutbahn. Zeitschr. f. experim. Pathol. u. Ther. 11. 1912. Kontroverse mit Roux, siehe R. Thoma, Virchows Archiv 207, 210. 1912. Die Gestalt der Gefäßlichtung bei der diffusen und knotigen Arteriosklerose. Virchows Archiv 216. 1914. - Die Strömung des Blutes in der Gefäßbahn und die Spannung der Gefäßwand. Beitr. z. pathol. Anat. u. z. allg. Pathol. 66. 1920. - Über die Intima der Arterien. Virchows Archiv 230. 1921. - Von älteren Arbeiten sei nur erwähnt: R. Thoma, Untersuchungen über die Histogenese und Histomechanik des Gefäßsystems. Stuttgart 1893. 
man behaupten, daß die allgemeinen Ergebnisse dieser Untersuchungen auch in den Einzelheiten vollinhaltlich mit dem tatsächlichen Geschehen in Übereinstimmung stehen.

$$
\begin{aligned}
& \text { 4. Arteriensystem vom Typus: } \varrho=\text { konstant und } \\
& \beta=\text { konstant. }
\end{aligned}
$$

Wenn bei dem normalen Wachstum ebenso wie bei den pathologischen Vorgängen der Radius der Gefäßlichtung größer oder kleiner wird, bis die Geschwindigkeit des Blutstroms in dem kleinen Abstande $\beta$ von der Gefäßwand den Wert $\varrho$ erreicht hat, so gewinnt $\varrho$ die Bedeutung des kritischen Wertes der Randstromgeschwindigkeit, bei welchem das zirkuläre Wachstum der Gefäßwand zum Stillstande gelangt. Das zirkuläre Wachstum der Gefäßwand wird dagegen fortschreiten, solange die Randstromgeschwindigkeit in dem Abstande $\beta$ von der Gefäßwand größer als $\varrho$ ist. Während des Wachstums nimmt, wie meine histomechanischen Untersuchungen im einzelnen begründet haben, die mittlere Durchflußmenge der Arterien stetig zu, weil diese mittlere Durchflußmenge abhängig ist von dem Wachstum der Capillarnetze der Organe. Durch die Vermehrung der mittleren Durchflußmenge aber wird die Stromgeschwindigkeit in dem Abstande $\beta$ von der Gefäßwand um ein geringes größer als der kritische Wert $\varrho$. Damit ist sodann die Bedingung gegeben, welche in einer an anderem Orte ${ }^{1}$ ) näher besprochenen Weise ein positives zirkuläres Wachstum der Gefäßwand, eine Größenzunahme von $R$ auslöst. Bei dem Schwund der Organe und bei vielen pathologischen Vorgängen dagegen wird die Randstromgeschwindigkeit in dem Abstande $\beta$ von der Gefäßwand kleiner als $\varrho$ und löst in diesem Falle eine Größenabnahme von $R$, ein negatives zirkuläres Wachstum der Gefäßwand aus. Daß dabei unter normalen und pathologischen Bedingungen die histologischen Vorgänge scheinbar etwas verschieden sein können, soll hier nicht erörtert werden. Die Einzelheiten finden sich in meinen früheren Publikationen ohne Schwierigkeit. Sie dürften auch dem Physiologen und Embryologen vieles Interessante bieten.

Mit Hilfe der früher gegebenen Gleichung 2 findet man sodann für ein solches Arteriensystem das Druckgefälle in den verschiedenen Arterien gleich

$$
\frac{d p}{d x}=\frac{4 \vartheta}{2 R \beta-\beta^{2}} \varrho
$$

Da jedoch $\beta$ nur eine sehr kleine Größe darstellt, kann man für alle Arterien, deren Radius größer als $1 \mathrm{~mm}$ ist, den Wert von $\beta^{2}$ vernach-

1) R. Thoma, Beitr. z. pathol. Anat. u. z. allg. Pathol. 66. 1920. 
lässigen gegenüber der Größe $2 R \beta$. In diesem Falle geht die Gleichung 7 über in

$$
\frac{d p}{d x}=\frac{2 \vartheta}{R \beta} \varrho
$$

Für den gegebenen Typus des Arteriensystems ist jedoch $\varrho$ und $\beta$ konstant und für Arterien von mehr als $1 \mathrm{~mm}$ Radius kann man auch $\vartheta$ als eine Konstante ansehen. Für diesen Fall folgt aus Gleichung 8, daß bei dem in Rede stehenden Arterientypus das Druckgefälle umgekehrt proportional dem Radius der GefäBlichtung ist. In Arterien von weniger als $1 \mathrm{~mm}$ Radius wird, wie die Gleichung 7 zeigt, das Druckgefälle etwas höher sein.

Nach den Gleichungen 7 und 8 steigt, wenn $\varrho$ und $\beta$ konstante Werte darstellen, das Druckgefälle in den kleinen Arterien in erheblichem Maße. Es erhebt sich daher die Frage, ob ungeachtet dieser Zunahme des Druckgefälles die Gleichungen 1 und 2 in Verbindung mit Gleichung 6 richtige Werte für die Durchflußmengen ergeben. Ich habe bei der ersten Behandlung dieser Gleichungen im Jahre 1910 angenommen, daß diese Frage zu bejahen ist, weil bei konstanter Randstromgeschwindigkeit in dem konstanten Abstande $\beta$ von der Gefäßwand kein Anlaß gegeben scheint zu der Annahme, daß die Verschiedenheiten des Druckgefälles Störungen bewirken könnten. Nach dem Inhalte des Abschnittes 2 scheint dieser Schluß auch heute noch gerechtfertigt zu sein. Nicht das ungleiche Druckgefälle, sondern die ungleiche Randstromgeschwindigkeit oder die ungleiche Breite der zellfreien Randzone dürfte bei den Viscosimeterversuchen die Störungen bewirken, welche Hess und Roth mann als die Folge der Verschiedenheiten des Druckgefälles betrachten. Doch ist in Ermangelung systematischer, auf diesen Punkt bezüglicher Untersuchungen zuzugeben, daß obige Entscheidung noch keine endgültige sein kann. Es wäre möglich, daß später noch kleine Korrekturen an den Durchflußmengen anzubringen sind.

Indessen steht man hier vor der Aufgabe, die Durchflußmengen empirisch zu prüfen und auf empirischem Wege die Genauigkeit der gegebenen Stromgleichungen nachzuweisen. Zu diesem Zwecke kann man zunächst die Breite $\not \beta$ der plasmatischen Randzone mit $0,01 \mathrm{~mm}$ in Rechnung stellen. Damit begeht man jedoch, wie es scheint, eine Willkürlichkeit und zwar aus verschiedenen Gründen. Erstens entzieht sich die Breite $\beta$ der plasmatischen Randzone in den größeren Arterien der direkten Beobachtung und zweitens stellt $\beta=0,01 \mathrm{~mm}$ den größten Wert dar, welcher in Ubbereinstimmung mit der Beobachtung für die kleineren Arterien gewählt werden kann. Er entspricht ungefähr dem Abstande des Schwerpunktes der äußersten Zellen des roten Axialstromes von der Gefäßwand. Würde man dagegen den Begriff der plasmatischen Randzone auf dasjenige Gebiet des Stromes besehränken, in welches 
bei der Beobachtung am lebenden Warmblüter in der Regel kein einzelner Teil der roten Zellen eintaucht, so würde $\beta$ ungefähr gleich $0,0035 \mathrm{~mm}$ $\mathrm{zu}$ veranschlagen sein. Indessen ist diese Willkürlichkeit ohne nennenswerte Bedeutung für die Berechnung der Durchflußmengen in den Arterien von mehr als $1,0 \mathrm{~mm}$ Radius. Es geht dies hervor aus dem Unstande, daß $\beta$ im Verhältnisse zu $R$ eine sehr kleine Größe darstellt. Dies ist in einfacher Weise auch auf numerischem Wege zu bestätigen.

Wenn man nach genauen Messungen den Radius der Lichtung der aufsteigenden Aorta des normalen Menschen am Schlusse des Wachstums gleich 11,2 mm annimmt und zugleich die Durchflußmenge $W$ derselben nach den Üntersuchungen der besten Autoren mit $82500 \mathrm{cmm}$ in der Sekunde in Rechnung stellt, so findet man mit Hilfe der Gleichung 1

$$
W=\frac{\pi}{2}\left[R^{2}+(R-\beta)^{2}+\frac{(R-\beta)^{4}}{2 n \beta\left(R-\frac{\beta}{2}\right)}\right] \varrho
$$

und wenn man zugleich $\beta=0,01 \mathrm{~mm}$ und den entsprechenden Wert von $n$ aus der Hyperbelgleichung 6 einstellt

$$
\varrho=1,27776 \mathrm{~mm} \text { in der Sekunde. }
$$

Sodann ergibt sich mit Hilfe derselben Gleichungen 1 und 6 für diesen Wert von $\varrho$ die Durchflußmenge einer Arterie von $2,0 \mathrm{~mm}$ Radius gleich $438,1 \mathrm{ccm}$ in der Sekunde und die Durchflußmenge einer Arterie von $1,0 \mathrm{~mm}$ Radius gleich $50,85 \mathrm{cmm}$ in der Sekunde.

Hätte man dagegen die Breite $\beta$ der plasmatischen Randzone gleich $0,0035 \mathrm{~mm}$ angenommen, so würde man, wiederum unter der Voraussetzung, daß die Durchflußmenge der Aorta ascendens $82500 \mathrm{cmm}$ betrage, auf dem gleichen Wege den viel kleineren Wert

$$
\varrho=0,44808 \mathrm{~mm} \text { in der Sekunde }
$$

erhalten haben. Die Durchflußmenge in der Arterie von 2,0 mm Radius aber würde gleich $433,4 \mathrm{cmm}$ in der Sekunde und in der Arterie von 1,0 mm Radius gleich $49,45 \mathrm{cmm}$ in der Sekunde gefunden werden. Der sich ergebende Fehler ist jedoch von keiner großen Tragweite. Für $R=3,0 \mathrm{~mm}$ und mehr würde er noch erhehlich kleiner ausfallen.

Wenn es aber für die Berechnung der Durchflußmengen der großen Arterien bis herab zu Arterien von 1,0 mm Radius von keiner wesentlichen Bedeutung ist, ob $\beta$ etwas größer oder kleiner gewählt wird, so fällt zunächst das große Bedenken, welches sich an die Tatsache knüpft, daß die Breite der plasmatischen Randzone sich in den großen Arterien der direkten Beobachtung entzieht. Für die Arterien von weniger als $1,0 \mathrm{~mm}$ Radius aber darf man $\beta$ nicht kleiner als $0,01 \mathrm{~mm}$ ansetzen, weil für kleinere Werte von $\beta$ die Randstromgeschwindigkeit $\varrho$ in dem Abstande $\beta$ von der Gefäßwand so klein wird, daß sie in den kleinen, der mikroskopischen Untersuchung zugängigen Arterien in einen auffallenden Widerspruch mit der Beobachtung gerät. Die Randstromgeschwindigkeit $\varrho$ wird bei der mikroskopischen Untersuchung direkt an der Bewegung der Zellen erkennbar, welche die Oberfläche des roten 
Axialstromes bilden. Dabei sei mir die Bemerkung gestattet, daß ich hier die Verhältnisse, wie sie beim Warmblüter zu beobachten sind ${ }^{1}$ ), im Auge habe. Die Randstromgeschwindigkeit ist in den Arterien des Frosches erheblich geringer als in den Arterien des Hundes und des Kaninchens, jedoch immer noch groß genug, um diese Bedenken zu rechtfertigen.

Hier machen sich bereits Umstände bemerklich, welche es zweifelhaft erseheinen lassen, ob dieser Arterientypus ( $\beta=$ konstant und $\varrho=$ konstant) in dem menschlichen Arteriensystem verwirklicht ist. Diese Zweifel werden sich später als berechtigt erweisen. Vorläufig können sie noch nicht als ausschlaggebend betrachtet werden und außerdem erscheint es zweckmäßig, die Untersuchung eines Arteriensystems von dem gegebenen Typus zu Ende zu führen.

Damit gelangt man dazu, zunächst die Werte $\beta=0,01 \mathrm{~mm}$ und $\varrho=1,278 \mathrm{~mm} /$ Sek. zur Grundlage der weiteren Untersuchung zu nehmen, indem man zugleich beachtet, daß entscheidende Schlußfolgerungen bezüglich der Durchflußmengen nur gezogen werden können aus der Untersuchung von Arterien von mehr als 1,0 mm Radius. In der Folge erscheint daher $\varrho=1,278 \mathrm{~mm} / \mathrm{Sek}$. als der kritische Wert der Randstromgeschwindigkeit, weil den Voraussetzungen gemäß das positive oder negative, zirkuläre Wachstum der Arterienwand solange fortschreitet, bis dieser kritische Wert in allen Arterien erreicht ist. Die Eigenschaften eines solchen Arteriensystemes, die Durchflußmengen, die axialen Geschwindigkeiten, das Druckgefälle und die Verzweigungsexponenten der verschiedenen Arterien habe ich früher tabellarisch zusammengestellt ${ }^{2}$ ), allerdings unter Benützung nicht völlig mit Gleichung 6 übereinstimmender Werte von $n$. Die Hyperbel, welche dieser Gleichung zugrunde liegt, war damals nur auf graphischem Wege, somit etwas weniger genau gefunden worden.

Diese Zusammenstellung geschah indessen damals durchaus nicht, um die Richtigkeit der von mir aufgestellten Stromgleichungen zu beweisen. Diese stehen durchaus auf eigenen Füßen. Es war nur meine Absicht zu zeigen, daß die gegebenen Voraussetzungen ( $\beta=$ konstant, $\varrho=$ konstant) zu keinen durchaus unwahrscheinlichen Werten führen. Dieses Ziel glaube ich erreicht zu haben. $\mathrm{Hess}^{3}$ ) stimmt jedoch mit diesem Ergebnisse nicht überein, indem er, von anderen Gesichtspunkten ausgehend, einen scheinbar neuen Typus aufstellt. Dabei begegnet ihm allerdings das Mißgeschick, daß sein scheinbar neuer Typus mit dem hier besprochenen Typus bis auf Größen höherer Ordnung genau identisch ist.

1) R. Thoma, Virchows Archiv r4. 1878.

$\left.{ }^{2}\right)$ R. Thoma, Dtsch. Arch. f. klin. Med. 99, 616. 1910. Tabelle IX.

s) W. R. Hess, Arch. f. d. ges. Physiol. 168. 1817. 
Offenbar unter dem Einfluß der Lehren von Roux sucht Hess zunächst nach einer Zweckmäßigkeit und findet dieselbe durch den Nachweis, daß bei gleichbleibender Länge und gleichbleibendem Voluminhalt einer verzweigten Arterienbahn die Widerstände für den Blutstrom ihren kleinsten Wert besitzen, wenn für die symmetrische Bifurkation der Arterien

$$
q_{n}=q_{a} \sqrt[3]{2}
$$

Diese Gleichung stellt einen bestimmten Bautypus eines Arteriensystemes dar, der hier zu analysieren ist. In der Gleichung 9 bezeichnet $q_{a}$ den Querschnitt des Stammes einer Verzweigung, den man auch schreiben kann $\pi R^{2}$, wenn der Radius des Stammes gleich $R$ gesetzt wird. Sodann gibt $q_{n}$ die Summe der Querschnitte der beiden zugehörigen, unter sich gleichgroßen Zweige. Nimmt man den Radius dieser Zweige gleich $r$, so wird $q_{n}=2 \pi r^{2}$. Dabei geht die Gleichung 9 über in

$$
2 r^{3}=R^{3} \text {. }
$$

Die Größe, die ich früher als Verzweigungsexponent bezeichnet habe, ist gleich 3.

Nach dem Gesetze von Hagen ist sodann die Durchflußmenge $W$ des Stammes vom Radius $R$ gleich

$$
W=\frac{\pi}{8 \eta} R^{4} \frac{d P}{d X}
$$

und die Durchflußmenge $w$ jedes der beiden $Z_{w}$ eige gleich

$$
w=\frac{\pi}{8 \eta} r^{4} \frac{d p}{d x}
$$

Sodann muB $W=2 w$ sein, woraus folgt, wenn man zugleich die Gleichung 10 berücksichtigt

$$
R \frac{d P}{d X}=r \frac{d p}{d x}
$$

oder, wenn $k$ eine Konstante darstellt,

$$
\frac{d P}{d X}=\frac{k}{R} \quad \text { und } \quad \frac{d p}{d x}=\frac{k}{r}
$$

Die Bedingung, welche in der von Hess aufgestellten Gleichung 9 enthalten ist, findet somit Erfüllung, wenn das Druckgefälle im Stamm und in den zugehörigen $Z_{w}$ eigen einer symmetrischen Bifurkation umgekehrt proportional dem GefäBradius ist.

Nach den Entwicklungen von Wiedemann kann man sodann die Stromgeschwindigkeiten $U$ und $u$ in dem konstanten Abstande $\alpha$ von der Wand schreiben für den Stamm

und für die Zweige

$$
U=\frac{1}{4 \eta}\left(2 R \alpha-\alpha^{2}\right) \frac{d P}{d X}
$$

$$
u=\frac{1}{4 \eta}\left(2 r \alpha-\alpha^{2}\right) \frac{d p}{d x}
$$

Setzt man sodann für das Druckgefälle die Werte aus den Gleichungen 12 ein, so folgt

$$
\begin{aligned}
U & =\frac{1}{4 \eta}\left(2 \alpha-\frac{\alpha^{2}}{R}\right) k \\
u & =\frac{1}{4 \eta}\left(2 \alpha-\frac{\alpha^{2}}{r}\right) k
\end{aligned}
$$

Wenn nun $\alpha$ klein ist im Verhältnis zu den Radien der Gefäßlichtungen, so wird $U$ und $u$ zu der Randstromgeschwindigkeit $U_{1}$ und $u_{1}$ in dem Abstande $\alpha$ 
von der Wand und man erhält, da das zweite Glied unter der Klammer als verschwindend klein angesehen werden kann im Verhältnis zu $2 \alpha$, 'das Ergebnis

$$
U_{1}=u_{1}=\text { konstant und } \alpha \text { konstant. }
$$

Hier wurde das Blut als eine homogene Flüssigkeit behandelt, wie dies auch von Hess geschah. Zu genau dem gleichen Ergebnisse gelangt man jedoch auch, wenn man zwischen dem plasmatischen Randstrom und dem roten Axialstrom unterscheidet. Sowie $\frac{d p}{d x}=\frac{k}{R}$ gesetzt wird, folgt auch aus der zu Eingang gegebenen Gleichung 2 , daß die Randstromgeschwindigkeit $\varrho$ konstant wird für alle Werte des Gefäßradius, denen gegenüber $\beta$ sehr klein erscheint. Aus der Gleichung 8 aber kann man den Wert der Konstanten $k$ finden

$$
k=\frac{2 \vartheta \underline{\varrho}}{\beta}
$$

Dieser Wert ist konstant, solange $\vartheta$ als Konstante betrachtet werden kann. Die Gültigkeit dieser Erörterungen erscheint somit beschränkt auf das Gebiet, für welches das Gesetz von Hagen als maßgebend angesehen werden kann, also auf Arterien, deren Radius $1 \mathrm{~mm}$ übersteigt.

Innerhalb des Geltungsbereiches des Gesetzes von Hagen, welche Beschränkung auch von Hess vorausgesetzt wird, ist somit die von Hess aufgestellte Gleichung 9 gleichbedeutend mit der Bedingung $(\varrho=$ konstant, $\beta=$ konstant). Hess ist durch seine Erwägungen zu dem von ihm verworfenen Arterientypus gelangt, welcher durch die Bedingung einer konstanten Randstromgeschwindigkeit gekennzeichnet wird. Zu einer Erklärung der Entwicklungsmechanik und der Wachstumsvorgänge dürfte jedoch die von ihm gemachte Voraussetzung einer Konstanz der Länge und des Voluminhaltes des Arteriensystemes wenig geeignet sein, während man sich in sehr einfacher Weise eine Vorstellung darüber bilden kann, daß die Arterienwand die Geschwindigkeit des strömenden Blutes empfindet und unter normalen wie unter pathologischen Bedingungen auf diese Empfindung durch ein positives oder negatives zirkuläres Wachstum reagiert, bis die Randstromgeschwindigkeit $\varrho$ ihren kritischen Wert erreicht.

Über die Einzelheiten dieses Vorganges, über seine Beziehungen zum Tonus der Gefäßwand und zu den Materialspannungen und über die zugehörigen Wachstumserscheinungen in den Geweben habe ich mich unlängst an anderen Orten ${ }^{1}$ ) genügend ausgesprochen. Die Reaktion der Gefäßwand auf die Geschwindigkeit der Randzonen des Blutstromes ist eine besondere vielleicht vom Parablasten herstammende Eigenschaft, welche die Gefäßwand von allen anderen Organen unterscheidet.

Begnügt man sich dagegen, mit Roux die ZweckmäBigkeit einer Einrichtung durch eine Anpassung und die Anpassung durch eine Anpassungsfähigkeit der Gewebe und Organe zu erklären, ohne an dieser

1) R. Thoma, Beitr. z. pathol. Anat. u. z. allg. Pathol. 66. 1920. - Virchows Archiv 230. 192]. 
Tautologie Anstoß zu nehmen, so wird man auch die Entwicklung und das Wachstum der Arterien in ihren allgemeinen Umrissen bequem zu erklären vermögen durch Schlußfolgerungen, welche von einer Konstanz der Länge und des Voluminhaltes der*Arterien ausgehen.

Es fragt sich jedoch, ob die Bedingung ( $\varrho=$ konstant, $\beta=$ konstant) und damit auch die von Hess in Gleichung 9 aufgestellte Bedingung mit den tatsächlichen Verhältnissen einigermaßen genau übereinstimmt. $\mathrm{Zu}$ diesem Zwecke wende ich mich der Frage zu, in welcher Weise man diese Bedingung auf empirischem Wege genauer prüfen kann.

$$
\begin{gathered}
\text { 5. Arteriensystem vom Typus: } \\
\varrho=0,362+1,019^{(11,2-R)^{2}} \text { und } \beta=0,01 \mathrm{~mm} .
\end{gathered}
$$

Die eingangs besprochene Gleichung 1 gibt die mittlere Durchflußmenge $W$ einer Arterie als Funktion des Radius $R$ in Vielfachen der Randstromgeschwindigkeit $\varrho$

$$
W=\frac{\pi}{2}\left[R^{2}+(R-\beta)^{2}+\frac{(R-\beta)^{4}}{2 n \beta\left(R-\frac{\beta}{2}\right)}\right] \varrho
$$

Zugleich ist es selbstverständlich, daß die Durchflußmenge $W$ jedes Arterienstammes gleich ist der Summe der Durchflußmengen $w_{1}+w_{2}$ $+w_{3}+\ldots$ seiner Zweige, so daß

$$
W=w_{1}+w_{2}+w_{3}+\ldots
$$

Wenn man sodann für eine größere Zahl von Arterienverzweigungen, welche zuvor unter der Wahrung der nötigen Vorsichtsmaßregeln, bei konstantem, dem Blutdrucke entsprechenden Drucke mit Paraffin injiziert wurden ${ }^{1}$ ), die Radien der Lichtung ausmißt, so ist man offenbar in der Lage, mit Hilfe der Gleichung 1 für jeden Arterienstamm und für seine Zweige die Durchflußmengen in Vielfachen von $\varrho \mathrm{zu}$ berechnen, um diese Werte sodann in die Gleichung 13 einzusetzen. Damit findet man die Randstromgeschwindigkeit $\varrho$, gleichviel ob diese eine konstante oder eine variable Größe ist.

Obigen Erörterungen entsprechend gewinnt man zuverlässige Resultate nur, wenn die Radien der Lichtung der gemessenen Arterien größer als $1 \mathrm{~mm}$ sind. $\mathrm{Da} ß$ zugleich die Wahl der Größe $\beta$ innerhalb der oben gegebenen Grenzen von keiner wesentlichen Bedeutung ist, wurde gleichfalls oben besprochen. Ich habe $\beta=0,01 \mathrm{~mm}$ angenommen. Es wäre jedoch auch denkbar, wenn man über eine große Zahl von Beobachtungen verfügt, den Wert von $\beta$ aus diesen zu berechnen. Doch dürfte dies auf rechnerische Schwierigkeiten stoßen und außerdem wesentlich genauere Beobachtungen voraussetzen.

Die Genauigkeit und Zuverlässigkeit der Resultate ist indessen größer, als man vielleicht anzunehmen geneigt ist, weil geringe aber proportionale Anderungen der

1) Die etwas komplizierte Technik der Injektion. und der Ausmessung der Arterien, sowie die gewonnenen Resultate finden sich ausführlich dargestellt in den Beitr. z. pathol. Anat. u. z. allg. Pathol. 66. 1920. 
Radien des Stammes und der zugehörigen Zweige, wie sie bei manchen Teilen des technischen Verfahrens zu gewärtigen sind, zwar die Durchflußmengen in nicht unerheblicher Weise ändern, jedoch den Vergleich der Durchflußmengen des Stammes mit den Durchflußmengen seiner Zweige, wie er durch die Gleichung 13 herbeigeführt wird, nur in sehr geringem Grade stören.

Für die Werte von $n$ hatte ich bei dieser Untersuchung noch die früher auf graphischem Wege gewonnenen Zahlen benützt. Ich werde die dadurch bedingten Abweichungen erwähnen. Sie sind im ganzen unerheblich.

Die weitere Untersuchung zeigte zunächst, daß es ganz ausgeschlossen ist, die Randstromgeschwindigkeit $\varrho$ als eine konstante Größe anzusehen. Die Summen der Durchflußmengen der Zweige werden für $\varrho=$ konstant immer viel kleiner gefunden als die Durchflußmenge des Stammes. Die Differenz als Fehler betrachtet betrug in der Mehrzahl der Fälle mehr als 27\% der Durchflußmenge des Stammes ${ }^{1}$ ). Die Durchflußmengen der Stämme wurden jedoch ziemlich genau gleich der Summe der Durchflußmengen der zugehörigen Zweige, als ich $\varrho$ als eine Funktion von $R$ betrachtete und durch ein Annäherungsverfahren fand:

$$
\varrho=0,362+1,019^{(11,2-R)^{2}} \mathrm{~mm} / \text { Sek. }
$$

Bei dem Gebrauch dieser Gleichung erreichte der größte Fehler bei der Berechnung der Durchflußmengen nur 5,5\% der Durchflußmenge des Stammes und die Fehler waren, wie dies bei zufälligen Fehlern immer der Fall ist, abwechselnd positiv und negativ. Die Gleichung 14 scheint somit der Wahrheit ziemlich nahezukommen.

Bei der Aufstellung der Gleichung 14 bin ich wieder ausgegangen von dem anderweitig gewonnenen Ergebnisse, daß die mittlere Durchflußmenge der aufsteigenden Aorta $(R=11,2 \mathrm{~mm})$ in der Sekunde $82500 \mathrm{cmm}$ beträgt. Aus dieser Annahme folgte mit Hilfe der Gleichung 1 alsbald die Randstromgeschwindigkeit in der aufsteigenden Aorta gleich 1,362 mm, wobei die auf graphischem Wege gewonnenen Werte von $n$ Verwendung fanden und $\beta=0,01 \mathrm{~mm}$ angenommen war. Für die mittleren und kleineren Arterien aber ergab die Gleichung 14 allmählich größere Werte für die Randstromgeschwindigkeit. In den Arterien von $1 \mathrm{~mm}$ Radius wurde $\varrho$ annähernd gleich $7,4 \mathrm{~mm}$ in der Sekunde und in Arterien von $0,028 \mathrm{~mm}$ Radius gleich $10,8 \mathrm{~mm}$ in der Sekunde. Die Stromgeschwindigkeit in der Achse des roten Axialstromes aber betrug in der Aorta ascendens $418 \mathrm{~mm}$ in der Sekunde, nahm in den mittleren und kleinen Arterien allmählich ab und wurde in einer Arterie von $1 \mathrm{~mm}$ Radius gleich $201 \mathrm{~mm} / \mathrm{Sek}$. und in Arterien von $0,028 \mathrm{~mm}$ Radius gleich $11,0 \mathrm{~mm} /$ Sek.

1) Diese Fehler wären für ( $\varrho=$ konstant, $\beta=$ konstant) noch etwas größer geworden, wenn statt der auf graphischem Wege gewonnenen Hyperbelwerte von $n$ die aus der Hyperbelgleichung 6 hervorgehenden Werte von $n$ Verwendung gefunden hätten. 
Ich gehe an dieser Stelle auf die sehr kleinen Arterien ein, obgleich für diese die verwendeten Werte von $n$ und manche andere Einzelheiten als weniger genau gelten müssen, weil die Stromgeschwindigkeiten nur in diesen sehr kleinen Arterien einer empirischen Nachprüfung durch das Mikroskop unterzogen werden können. Die unter der Leitung von Hürthle ausgeführten Stromuhrversuche, die ich gleichfalls berücksichtigt habe ${ }^{1}$, liefern allerdings annähernd stimmende Resultate. Sie sind jedoch nicht genau genug, um über die verschiedenen Bautypen des Arteriensystemes eine Entscheidung zu bringen. Dies beruht hauptsächlich auf dem Umstande, daß bei diesen Stromuhrversuchen die Gefäßdurchmesser nicht hinreichend genau gemessen werden können. Die lichte Weite der bloßgelegten Teile der Arterien dürfte infolge von Störungen der Innervation immer abnorme Werte ergeben.

Wenn man bei dem Schlage eines Metronoms oder eines Sekundenpendels den Blutstrom in den kleinen Arterien des Netzes oder des Mesenterium des Hundes beobachtet ${ }^{2}$ ), so wird man sich davon überzeugen können, daß die aus Gleichung 14 berechneten Geschwindigkeiten an der Oberfläche des roten Axialstromes annähernd wenigstens mit der Wirklichkeit in Ubereinstimmung stehen. Ich war in der letzten Zeit nicht mehr in der Lage, diese Beobachtungen zu wiederholen. Nachdem ich mich jedoch früher jahrelang in aufmerksamster Weise mit dem Blutstrom der Säugetiere beschäftigt habe, glaube ich diese Behauptung verantworten zu können. Sie findet in gewissem Sinne eine Bestätigung durch die kinematographischen Untersuchungen von Hürthle ${ }^{3}$ ), welcher in einer Arterie von $0,015 \mathrm{~mm}$ Radius beim Frosch Stromgeschwindigkeiten von $5 \mathrm{~mm}$ in der Sekunde nachgewiesen hat. Denn es kann kein Zweifel darüber bestehen, daß die Stromgeschwindigkeiten in den kleinen Arterien des Frosches erheblich kleiner sind als in den kleinen Arterien der Säugetiere.

Dabei erscheint es als unwesentlich, ob Hürthle die Stromgeschwindigkeiten in der Achse oder an der Oberfläche des roten Axialstromes kinematographisch gemessen hat. Denn Rechnung und Beobachtung ergeben in übereinstimmender Weise, daß in den verwende-

1) R. Thoma, Virchows Archiv 204, 20. 1911.

2) Über die Technik solcher Versuche vgl. R. Thoma, Virchows Archiv $\boldsymbol{y 4}$. 1878. Kaninchen sind zu diesen Versuchen wenig brauchbar, weil bei einigermaßen ausgiebiger Eröffnung der Bauchhöhle der Blutdruck erheblich absinkt, indem eine hochgradige Hyperämie der vorgelagerten Eingeweide eintritt. Diese Hyperämie ist mit einer starken Beschleunigung des arteriellen Stromes in den vorgelagerten Teilen verbunden. Übrigens erfordert es auch längere Erfahrung, bis man beim Hunde unter solchen Bedingungen ein Urteil über die normale Stromgeschwindigkeit in den Arterien gewinnt. Die vorgelagerten Teile sollten mit körperwarmer 0,9 proz. Kochsalzlösung irrigiert werden und auf einem entsprechend erwärmten, durchsichtigen Objektträger liegen.

3) K. Hürthle, Arch. f. d. ges. Physiol. 162. 1915. 
ten, kleinen Arterien die Stromgeschwindigkeiten in der Achse und an der Oberfläche des roten Axialstromes annähernd genau gleich groß sind. Zugleich beweisen die großen Stromgeschwindigkeiten in den kleinen Arterien, da $\beta$ die Bedingung ( $\varrho=$ konstant, $\beta=$ konstant), welche für den ersten Typus des Arteriensystems gilt, der Wirklichkeit nicht entspricht. Für diesen ersten Typus war die Randstromgeschwindigkeit überall gleich $1,278 \mathrm{~mm} / \mathrm{sek}$., wenn $\beta=0,01 \mathrm{~mm}$ angenommen wird, also in den kleinen Arterien viel zu gering. An Hürthle jedoch, der über Hilfsarbeiter und über die nötigen Apparate verfügt, richte ich die Bitte, auch die Stromgeschwindigkeiten in den kleinen Arterien der Säugetiere kinematographisch festzustellen. Eine genauere Kenntnis dieser Stromgeschwindigkeiten wäre, wie man bemerkt, von großer Bedeutung für die Beurteilung der Kreislaufsvorgänge beim Menschen.

Aus der zu Eingang gegebenen Gleichung 2 folgt sodann für den hier in Rede stehenden, zweiten Bautypus des Arteriensystemes das Druckgefälle

$$
\frac{d p}{d x}=\frac{4 \vartheta}{\left(2 \beta-\frac{\beta^{2}}{R}\right)} \cdot \frac{\varrho}{R}
$$

und für größere Arterien, für welche man ohne wesentliche Ungenauigkeit das zweite Glied des in der Klammer stehenden Ausdruckes weglassen kann

$$
\frac{d p}{d x}=\frac{2 \vartheta}{\beta} \cdot \frac{\varrho}{R}
$$

Setzt man sodann aus der Gleichung 14 den variablen Wert von $\varrho$ ein, so überzeugt man sich leicht davon, daß in Utbereinstimmung mit den Lehren der Physiologie das Druckgefälle in den großen und mittleren Arterien sehr gering ist, während es in den kleinen und kleinsten Arterien eine beträchtliche Höhe erreicht. In der Aorta ascendens ( $R=11,2 \mathrm{~mm})$ wird rechnungsgemäß auf jeden Millimeter Strombahnlänge $0,000225 \mathrm{~mm}$ $\mathrm{hg}$ verbraucht. In einer Arterie von $1 \mathrm{~mm}$ Radius hat sich dieser Verbrauch für jeden Millimeter der Strombahnlänge auf $0,0140 \mathrm{~mm} \mathrm{hg}$ gesteigert und in einer Arterie von $0,028 \mathrm{~mm}$ Radius würde er für jeden Millimeter Strombahnlänge $1,246 \mathrm{~mm}$ hg betragen.

Bei der Berechnung des Druckgefälles $\frac{d p}{d x}$ wurde hier berücksichtigt, daß der Viseositätskoeffizient $\vartheta$ des Blutplasmas in den kleinen Arterien zunimmt. Oben hatte ich für den Viscositätskoeffizienten des Blutplasmas des Pferdes zwei Gleichungen abgeleitet, welche sich auf die Temperaturen von $32,2^{\circ}$ und $40,4^{\circ} \mathrm{C}$ bezogen. Wenn man annimmt, daß für normale Körpertemperatur die Werte von $\vartheta$ annähernd in der Mitte zwischen den Angaben dieser beiden Gleichungen liegen und wenn man sodann diese Mittelwerte, mangels genauerer Bestimmungen der Viscosität des menschlichen BIutplasmas auch für den Menschen gelten läßt, so kommt noch in Betracht, daß diese Werte im Zentimeter-, Gramm., Sekunden- 
system ausgedrückt sind. Wird, wie es hier geschieht, der Druck nicht in Dynen, sondern in Millimetern hg gegeben, so müssen die gewonnenen Zahlen noch durch 1330 geteilt werden und man erhält

$$
\vartheta=0,000009233+\frac{0,0000001117}{R}
$$

wobei der Druck in Millimetern hg und der Radius $R$ der Gefäßlichtung in Millimetern in Rechnung zu stellen ist. Diese Werte von $\vartheta$ haben hier Verwendung gefunden. Sie wurden vierstellig gegeben, damit die Rechnung keine weiteren Fehler in diese Erwägungen bringen kann. Doch wird man.sich nicht täuschen lassen über die bestehenden Ungenauigkeiten. Vor allem fehlen uns genauere Viscositätsbestimmungen des menschlichen Blutplasmas und des menschlichen Blutes für die verschiedenen Werte von $R$. Diesen Viscositätsbestimmungen sollte allerdings eine systematische Prüfung der im Abschnitt 2 besprochenen Fragen vorangehen.

Die reziproken Werte der soeben im Haupttext für das Druckgefälle gegebenen Zahlen sind gleich den in Millimetern ausgedrückten Strombahnlängen, für welche jeweils $1 \mathrm{~mm}$ hg verbraucht wird. Sie betragen für die Aorta ascendens $4445 \mathrm{~mm}$, für eine Arterie von $1 \mathrm{~mm}$ Radius annähernd $71,5 \mathrm{~mm}$ und für die Arterie von $0,028 \mathrm{~mm}$ Radius annähernd $0,803 \mathrm{~mm}$, in annähernder Übereinstimmung mit den früher (Beitr. z. pathol. Anat. u. z. allg. Pathol. 66. 1920) gegebenen Zahlen. Die Unterschiede sind nur in den kleinsten Arterien etwas beträchtlicher, infolge der etwas höheren, in den kleinen Arterien zunehmenden Werte von $\vartheta$, die hier benützt wurden.

Es gibt noch einen anderen Weg, um den Inhalt der Gleichung 14 einer ziemlich strengen Prüfung zu unterziehen. Wenn man mit Hilfe der Gleichungen 1 und 14 die Durchflußmengen der zahlreichen größeren und kleineren Zweige der Aorta berechnet, so stimmt die Summe dieser Durchflußmengen in befriedigender Weise mit der Durchflußmenge der aufsteigenden Aorta überein, wie ich an einem anderen Orte ${ }^{\mathbf{1}}$ ) ausführlich nachgewiesen habe. Diese Beweisführung wäre meines Erachtens ausschlaggebend, wenn etwas genauere Zahlen für die Radien aller Zweige der Aorta vorliegen würden.

Immerhin berechtigt das gewonnene Ergebnis zusammen mit der obigen Untersuchung der einzelnen Verzweigungsstellen zu dem Schlusse, daß die Gleichungen 1 und 14

$$
\begin{aligned}
& W=\frac{\pi}{2}\left[R^{2}+(R-\beta)^{2}+\frac{(R-\beta)^{4}}{2 n \beta\left(\mathrm{R}-\frac{\beta}{2}\right)}\right] \varrho \\
& \varrho=0.362+1,019^{(11,2-R)^{2}} \mathrm{~mm} / \mathrm{sek} .
\end{aligned}
$$

eine annähernd richtige Vorstellung über die mittleren Durchflußmengen der Arterien des erwachsenen Menschen gewähren, wenn man die seinerzeit auf graphischem Wege gewonnenen und auch tabellarisch zusammengestellten ${ }^{2}$ ) Werte für den Viskositätsfaktor $n$ benützt und zugleich $\beta=0,01 \mathrm{~mm}$ annimmt.

1) R. Thoma, Beitr. z. pathol. Anat. u. z. allg. Pathol. 66. 1920.

2) R. Thoma, Dtsch. Arch. f. klin. Med. 99, 588, 616. 1910.

Pflügers Archiv f. d. ges. Physiol. Bd. 189. 
Die Zahl der Gefäßverzweigungen, auf deren Untersuchung dieses Ergebnis beruht, ist noch nicht sehr groß, was sich durch die große Umständlichkeit der Messung des Gefäßradius und durch die sehr zeitraubenden Ausrechnungen erklärt. Ich habe daher noch eine weitere Reihe von Arterienverzweigungen des Menschen injiziert und bin mit der Ausmessung derselben beschäftigt. Dabei erscheinen, wie ich später berichten werde, die vorstehenden Untersuchungen im wesentlichen bestätigt zu werden.

Zum Schlusse sei mir noch gestattet, auf die bemerkenswerte Tatsache hinzuweisen, daß sehr gegen mein Erwarten die Randstromgeschwindigkeit $\varrho$ nicht als eine Konstante, sondern als eine Exponentialfunktion von $R$ gefunden wurde. Dieses Ergebnis steht in einer bedeutsamen Beziehung zu den Gesichtspunkten, mit welchen ich an diese Untersuchung herantrat. Eine große Zahl anatomischer und experimenteller Erfahrungen an gesunden und kranken Blutgefäßen des Menschen und der Tiere hatte zu dem Schlusse genötigt, daß die Gefäßwand in irgendwelcher Weise die Geschwindigkeit der Randzonen des Blutstromes empfindet und auf diese Empfindung während des ganzen Lebens durch ein positives oder negatives Wachstum des Gefäßumfanges und somit auch des Gefäßradius reagiert in der Weise, daB eine bestimmte Randstromgeschwindigkeit $\varrho$ gewährleistet wird. Daß das Längenwachstum der Arterien anderen Gesetzen gehorcht, will ich hier nur nebenbei erwähnen. Ich glaube die Gesetze des Längenwachstums der Arterien an einem anderen Orte $^{1}$ ) mit vorläufig genügender. Genauigkeit festgestellt zu haben.

Wenn jedoch eine unbewußt sich vollziebende Empfindung das Wachstum des Gefäßradius beherrscht, so seheint auch für diese unbewußten Empfindungen das Gesetz von Fechner gültig zu sein. Durch einige Änderungen im Koordinatensystem, indem man den variablen Wert von $(\varrho-0,362)$ durch die neue Variable $v$ und den variablen Wert $(11,2-R)^{2}$ durch die neue Variable $\gamma$ ersetzt, geht, wenn zugleich die konstante Größe 1,019 mit $b$ bezeichnet wird, die Gleichung 14 über in

$$
v=b^{\gamma}
$$

Dieses aber ist der einfachste Ausdruck des Gesetzes von Fechner, in welchem $b$ eine empirisch zu bestimmende Konstante, $v$ den variablen Wert des Reizes und $\gamma$ den Erfolg des Reizes, also das Maß der Empfindung darstellt. Ich will diese Anschauung hier nur flüchtig angedeutet haben. Sie findet sich an dem soeben genannten Orte ausführlicher besprochen für die Gewebe, welche die Gefäßwand bilden.

1) R. Thoma, Beitr. z. pathol. u. z. allg. Pathol. 66. 1920. 